\title{
Asia Oceania Guidelines for the Implementation of Programs for Cervical Cancer Prevention and Control
}

\author{
Hextan Y. S. Ngan, ${ }^{1}$ Suzanne M. Garland, ${ }^{2}$ Neerja Bhatla, ${ }^{3}$ Sonia R. Pagliusi, ${ }^{4}$ \\ Karen K. L. Chan, ${ }^{1}$ Annie N. Y. Cheung, ${ }^{1}$ Tang-Yuan Chu, ${ }^{5}$ Efren J. Domingo, ${ }^{6}$ \\ You Lin Qiao, ${ }^{7}$ Jong Sup Park, ${ }^{8}$ Eng Hseon Tay, ${ }^{9}$ and Wisit Supakarapongkul ${ }^{10}$
}

${ }^{1}$ Department of Obstetrics and Gynaecology, Queen Mary Hospital, The University of Hong Kong, Pokfulam Road, Hong Kong

${ }^{2}$ Microbiological Research and Clinical Microbiology and Infectious Diseases, Royal Women's Hospital, Royal Children's Hospital, Department of Obstetrics \& Gynecology, University of Melbourne, Melbourne, VIC 3052, Australia

${ }^{3}$ Department of Obstetrics and Gynaecology, All India Institute of Medical Sciences, New Delhi 110 029, India

${ }^{4}$ Formerly Department of Immunization, Vaccines and Biologicals, World Health Organization, 1211 Geneva, Switzerland

${ }^{5}$ Center for Cervical Cancer Prevention, Department of Obstetrics and Gynecology, Graduate Institute of Clinical Medicine, Tzu Chi Medical Center, Tzu Chi University, Hualien 97004, Taiwan

${ }^{6}$ Department of Obstetrics and Gynecology, University of the Philippines College of Medicine, Manila, Philippines

${ }^{7}$ Department of Cancer Epidemiology, Cancer Institute/Hospital, Chinese Academy of Medical Sciences \& Peking Union Medical College, Beijing 100021, China

${ }^{8}$ Gynecologic Oncology, Department of Obstetrics and Gynecology, Seoul St. Mary's Hospital, The Catholic University of Korea School of Medicine, Seoul, Republic of Korea

9 Department of Obstetrics and Gynecology, KK Women's and Children's Hospital, Singapore 229899

${ }^{10}$ National Cancer Institute, Bangkok, Thailand

Correspondence should be addressed to Hextan Y. S. Ngan, hysngan@hkucc.hku.hk

Received 17 October 2010; Accepted 17 January 2011

Academic Editor: John D. Groopman

Copyright () 2011 Hextan Y. S. Ngan et al. This is an open access article distributed under the Creative Commons Attribution License, which permits unrestricted use, distribution, and reproduction in any medium, provided the original work is properly cited.

This paper aims to provide evidence-based recommendations for health professionals, to develop a comprehensive cervical cancer program for a clinic, a community, or a country. Ensuring access to healthcare is the responsibility of all societies, and the Asia Oceania Research Organisation in Genital Infections and Neoplasia (AOGIN) is committed to working collaboratively with governments and health professionals to facilitate prevention programs, to protect girls and women from cervical cancer, a disease that globally affects 500,000 and kills nearly 300,000 women annually, just over half of whom are in the Asia Oceania region. We share the vision that a comprehensive program of vaccination, screening, and treatment should be made accessible to all girls and women in the world. The primary purpose of these guidelines is to provide information on scientific evidence on the different modalities and approaches of cervical cancer prevention programs, for high resource and low resource settings. The secondary purpose is to provide an overview of the current situation of cervical cancer control and prevention in various Asian Oceania countries: their views of an ideal program, identified obstacles, and suggestions to overcome them are discussed.

\section{AOGIN Consensus Statement on Prevention and Control of Cervical Cancer}

Cervical cancer can be prevented in two ways:

(i) primary prevention, aimed at preventing or neutralizing the infection with human papillomavirus
(HPV), as for example through prophylactic HPV vaccination, and

(ii) secondary prevention, aimed at preventing precancerous lesions from progressing to invasive cancer through screening, early detection, diagnosis, and treatment, as clinically indicated. 
These are two distinct healthcare programs targeting women of different age groups, namely, vaccination of adolescent girls and young women, and commencement of screening of women aged 25 to 35 years. These two programs can be implemented in parallel or separately, depending on availability of suitable infrastructure and resources. Ideally, a combination of HPV vaccination programs together with effective screening and treatment for precancerous lesions has the greatest potential to significantly reduce the burden of cervical cancer.

The Asia Oceania Research Organization in Genital Infections and Neoplasia (AOGIN) endorses the World Health Organization (WHO) position [1] on primary prevention that "routine human papillomavirus (HPV) vaccination should be included in national immunization programs." The WHO statement is contingent on the provision that:

(i) prevention of cervical cancer and other related HPV diseases constitutes a public health priority,

(ii) vaccine introduction is programmatically feasible,

(iii) sustainable financing can be secured, and

(iv) cost-effectiveness of vaccine strategies in the country/region is considered.

As HPV vaccines are most efficacious in females who are naïve to vaccine-related HPV types, HPV vaccination programs should initially prioritize high coverage in the primary target population of girls aged 9-10 through 13 years.

AOGIN also endorses the principle that there should be cervical cancer screening procedures available to all women of appropriate age. This can be in the format of cervical cytology, visual inspection with acetic acid (VIA) or Lugol's iodine (VILI), or an HPV (high risk) DNA or RNA screen test.

\section{Introduction and Background}

Cervical cancer prevention and control is no longer just a dream. With the introduction of prophylactic vaccines against the oncogenic HPV 16 and HPV 18, with high coverage, we can prevent about $70 \%$ of cervical cancer worldwide if administered prior to exposure to infection. We can also expect the burden of cervical cancer to be greatly reduced within a couple of decades. With respect to secondary prevention, different screening methods, technologies, and strategies are developing to meet the challenges of improving their sensitivity, specificity, affordability, feasibility, acceptance, and compliance.

Each country in Asia Oceania has different burden of cervical cancer, economic status, political background, cultures, status of women's rights, and resources available for cervical cancer control. Thus, these guidelines serve two purposes: the first is to provide information on the current evidence on the different modalities and approaches, as well as to reinforce the recently published FIGO Global Guidance for Cervical Cancer Prevention and Control [2] with its

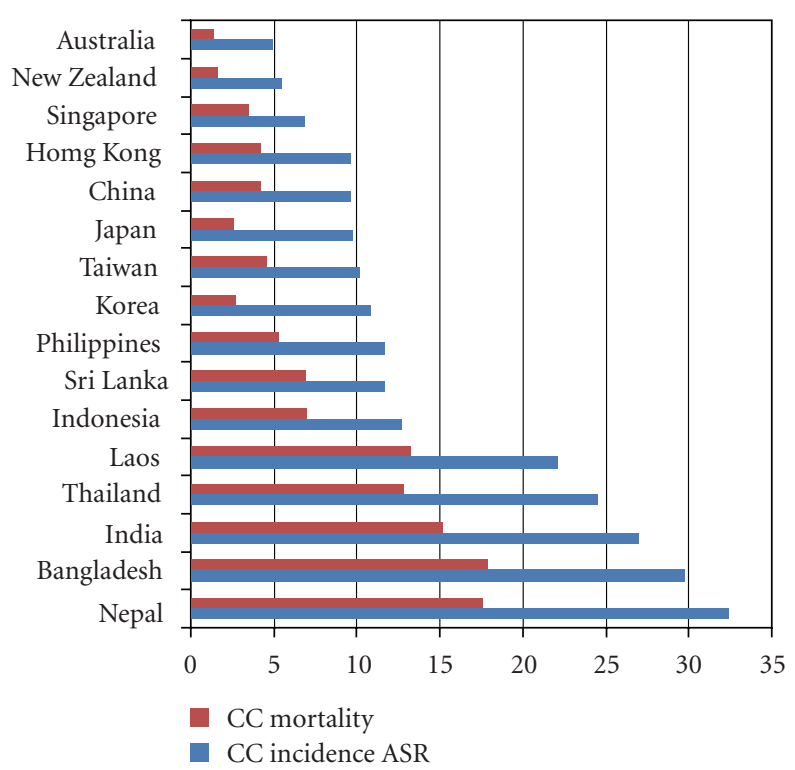

FIgURE 1: Incidence and mortality rates (per 100,000 women) due to cervical cancer (CC) in AOGIN country members (source Globocan at http://www.iarc.fr/).

comprehensive and practical review, as a source of reference. The second purpose is to provide contributions from various Asia Oceania countries on their current situation on cervical cancer control and prevention: what they consider as ideal ways to do it, obstacles they face, and suggestions to overcome them.

Although no country is the same, experience shows that problems encountered could be quite similar, and it is possible to learn from each other's experience into finding innovative solutions.

2.1. Epidemiology of Cervical Cancer. The incidence of cervical cancer among Asia Oceania countries varies from the lowest in Australia with age standardized rate (ASR) of 4.9 per 100,000 women to the higher ranges of 27 and 27.4 in India and Cambodia, respectively, to 28 in Mongolia, and 32 per 100,000 women in Nepal (Figure 1). Further, the ASR may vary within a country, particularly in large countries such as India and China. Moreover, if we consider analysis of the time trends of disease incidence, studies have suggested that cervical cancer incidence rates are increasing in recent generations, such as in China [3].

2.2. Variations in Economy. The wealth of countries within Asia Oceania varies widely not only among countries, but within a country. The largest economies in Asia in terms of nominal gross domestic product (GDP) [4] are Japan, China, and South Korea. Still, within one country there are also economic differences between urban and rural areas. For instance, in China there are provinces with a very poor economy, as compared to others. Similarly, in India, we see affluent cities with strong economy beside more rural states and poor provinces. 
2.3. Variety of Cultures. The countries of Asia Oceania represent an aggregate of cultural heritage of many different ethnicities, traditions, history, religions, and nationalities. Hence, certain clinical practices common in one place may not be acceptable in others. The social and moral norms may differ and may vary within countries and communities: hence the acceptance of specific clinical practices varies as well.

2.4. Variation in Recognition of Women's Rights. Women in many parts of Asia Oceania have variable legal rights, and some degree of discrimination against women is unfortunately relatively common. Unless social, cultural, as well as political will is coordinated to correct these inequalities, health care to women may continue to be perceived as a low priority, not just by providers and society but also by the women themselves.

\section{Primary Prevention Strategies}

The goal of primary prevention is to avoid exposure to the causative agent of diseases and thus to influence health outcomes. Primary prevention of cervical cancer can be achieved through prevention and control of genital HPV infections that cause the cancer. HPV is a ubiquitous virus; infections are very common and readily transmissible [5]. Health education campaigns geared at changing high risk sexual behaviours and targeting all STIs of public health significance, such as promoting delay in sexual debut and decrease in the number of sexual partners (or in their partners), as well as safe sexual practices can have some effect in reducing or preventing genital HPV infections. However, vaccination against HPV offers the greatest opportunity to prevent these infections on a large scale. Two prophylactic vaccines are now licensed and available to prevent HPV infections.

3.1. Prophylactic Vaccines. Currently two cervical cancer vaccines primarily aim for the prevention of HPV 16 and HPV 18 infections, as these two viruses account for about $70 \%$ of cervical cancer cases consistently worldwide [6]. Both vaccines induce humoral and cellular immunity and are designed for prophylactic use only, as they do not clear existing HPV infections nor treat established HPV-related diseases. Recently published studies report that these HPV $16 / 18$ vaccines also induce antibody responses to, and show partial efficacy against HPV types 31 and/or $45[7,8]$ which are phylogenetically similar to HPV-16 and - 18 , respectively. There are two key differences between the two available vaccines: the bivalent vaccine protects against the oncogenic types HPV 16 and HPV 18 while the quadrivalent vaccine also protects against two nononcogenic HPV types (HPV 6 and HPV 11) that cause the majority of genital warts [9]. The bivalent vaccine contains a novel adjuvant $\mathrm{ASO} 4$ which is considered responsible for a heighted immune response. The age group for which the HPV vaccines are licensed varies in different countries. The quadrivalent vaccine was originally licensed for use in females 9 to 26 years old, and has been extended in some countries up to 45 years of age. In some countries it is also licensed for use in males, including young boys. The bivalent vaccine is licensed for administration to females aged 10 to 45 years old. Recent data indicate sustained efficacy and immunogenicity of the vaccines up to almost 9 years [10]. Extensive phase 3 clinical trials and postmarketing surveillance continue to show that both HPV vaccines have high efficacy and good safety profiles, similar to other commonly administered vaccines. The characteristics, safety, immunogenicity and efficacy profile, and target population for the two available vaccines can also be found in the FIGO Global Guidance for Cervical Cancer Prevention and Control [2] (FIGO guide), Section 5.

3.2. Vaccination Programs. Merely the availability of effective and safe vaccines is not enough if the vaccines cannot be effectively delivered to target populations and with high coverage. The strategies of vaccination in low and high resource settings are discussed in the FIGO guidance, Section 6 [2]. In this context, schools seem to be promising venues for HPV immunization both in high- and lowresource countries, though in the latter the high dropout rates among girls may require additional strategies. It is encouraging to note that, for example, HPV vaccine coverage among school-aged female adolescents in Australia, as well as in their catch up program up to 26 years of age, has been estimated to reach up to $80 \%$. This has already translated into a statistically significant reduction in new cases of genital warts in women under 27 years of age in the order of $50 \%$, with a herd immunity effect on males [11]. Preliminary findings from demonstration projects conducted by PATH in India, Peru, Uganda, Mexico, and Vietnam suggest that a school-based approach can achieve coverage rates similar to those found in Australia. In Vietnam, community centre vaccine distribution had very high rates of compliance in a PATH-initiated demonstration project.

In Asia, the government of Bhutan has recently launched a national cervical cancer vaccination program through a collaboration with Merck Sharp \& Dohme (MSD) and the Australian Cervical Cancer Foundation (ACCF) [12]. Cervical cancer is the most common cancer among women in Bhutan, with annual incidence rate (ASR) of 20.4 per 100.000 women. As part of the 6-year program, Merck will provide quadrivalent (types 6, 11,16 and 18) Gardasil vaccine free to girls and young women in the country, in the first year, and at a concessional price for the remaining five years. Merck will also provide additional support for implementation of the program. ACCF, a charity whose mission is to minimize the incidence and burden of cervical cancer and related women's health issues on women, is supporting the national vaccination program by providing financial support to the Government of Bhutan to secure doses of Gardasil at the access price after the first year of the program is complete. The Royal Government of Bhutan is committed to ensuring sustainability of this program beyond the six-year partnership with Merck and ACCF. Bhutan is 
the first low-income country in the world to implement a national vaccination program with an HPV cancer vaccine.

The Malaysian government has announced that it will provide all 13-year-old girls with three doses of HPV vaccine to prevent cervical cancer. The program may cost the government RM150 million for 300,000 girls per year. The program will most probably be conducted in schools with the bivalent HPV vaccine (Cervarix) against HPV types 16 and 18, the more common types of HPV amongst Malaysian women. Pap smear tests will continue to be provided every three years in this country in which incidence of cervical cancer is 16.1 per 100,000 women, the second most frequent cancer for women in Malaysia after breast cancer. In Malaysia, women from the Chinese ethnic group are at higher risk with incidence rate of 23.2 for every 100,000 women, compared to 16.4 in ethnic Indian women and 8.2 in Malay women.

HPV vaccination has been named by the global alliance for vaccines and immunisation (GAVI) as a priority vaccine since 2008 which identified HPV vaccines as likely to have the biggest impact on the disease burden in developing countries. Both vaccines passed the WHO prequalification process. Support will depend on GAVI raising additional donor funds for countries wishing to introduce new vaccines [13]. We await the success of this initiative.

Program planners in many other countries will need to compare the costs of the two vaccines, depending on negotiations with manufacturers, and determine which one represents the better value based on available resources and current health priorities. As with other new health technologies, in many countries access to HPV vaccines through private physicians and clinics is far outpacing public sector programs. As a result, HPV vaccines are quickly becoming available to girls whose parents have the financial resources to cover the costs. However, this leaves the poor with no cover. It will be important to undertake countryspecific assessments of technologies and cost-effectiveness analyses, to make informed decisions about implementation of cervical cancer prevention programs.

It is important to appreciate that although the combination of vaccination and screening programs should be the aim for ideal cervical cancer prevention, countries lacking the necessary infrastructure for effective screening, followup, and treatment of women should feel encouraged to consider HPV vaccination as a highly feasible, sustainable and costeffective tool to tackle the issue, particularly in protecting younger generations, while building screening programs.

HPV vaccination is likely also to be beneficial to sexually active women over 25 years of age, who are at continuous risk of acquiring new HPV infections and developing cervical intraepithelial neoplasia (CIN) and cervical cancer. Clinical trial data show that both HPV vaccines are safe and immunogenic in women up to the age of 55 years, and are efficacious in women 24 to 45 years of age. The predicted reduction in cost-effectiveness of vaccination with increasing age, however, is likely to limit the implementation of routine vaccination beyond the late 20s. The priority of routine vaccination programs must be to target girls and young women, complemented with catch-up programs extending to ages 25-26 years, when resources allow. For sexually active women over the age of 25-26, HPV vaccination can be considered on an individual basis, as most women will have the potential to benefit from vaccination [14]. In addition, it has been shown that, in those who have been previously infected with vaccine-related HPVs (antibody positive, DNA negative to HPV 16 and/or 18), vaccinating these women has reduced HPV 16- and or HPV 18-related lesions.

\section{Secondary Prevention Strategies}

Once initial HPV infection has occurred, the goal of secondary prevention is to detect cervical disease in its earliest stages in women with persistent infection, before it progresses to invasive cancers. Since the 1950s, screening for cervical cancer based on detection of abnormal cervical cytology has been used worldwide. The impact of cervical cytology can be seen in some countries with successful, well-organised programs, with good coverage of the target population, plus with high-quality laboratory assurance. This is illustrated by the significant drop in incidence of cervical cancer in Australia, in women of all ages, decreasing from 12.7 to 4.9 per 100,000 , with mortality decreasing to $1.4 / 100,000$, one of the lowest worldwide (see below, Australia). However, examples of barriers to successful implementation of cervical cancer screening programs in the Asia Oceania region include lack of trained personnel for sample collection, processing, and testing, lack of adequately trained cytotechnicians, lack of high quality laboratory infrastructure for screening tests, and lack of subsequent follow-up colposcopy and treatment, due to both poor compliance as well as lack of health care providers, and not the least, the lack of awareness and support from politicians and government for financing such programs. Barriers encountered in low-resource settings can be found in the FIGO guidance, Sections 8-11 [2]. Hence, alternative methods for the secondary prevention of cervical cancer in sexually active women, mostly over 30 years of age, have been evaluated in numerous studies over the past $10-15$ years in different countries.

4.1. Screening Tests. Alternative screening methods have been sought, reported, and used in low-resource settings. These include visual inspection with acetic acid (VIA) and Lugol's iodine (VILI) and the single visit approach as discussed in FIGO guidance, Sections 8-9 [2]. Traditionally, a cervical smear is taken at the first visit and, if an abnormality is found, the women must return for further evaluation and at that point, diagnostic workup or treatment will be scheduled as required. In contrast, in the single visit approach, a screening test such as VIA provides rapid results, and treatment, usually cryotherapy, is made available to women with abnormal tests at the same visit, while a few women who need further treatment are referred elsewhere as appropriate. This approach is often referred to as the "Screen and Treat" or "See and Treat" approach.

The procedure involves applying 3-5\% freshly prepared acetic acid to the cervix and observing after one minute. 
However, acetowhite staining is not specific for CIN, being also able to stain mucosal ulceration due to other causes, for example, herpes, and even immature squamous metaplasia may appear acetowhite. Thus, up to $15-18 \%$ of women in any population may screen positive, as the specificity of VIA is lower than Pap. This leads to high referral rates for further evaluation or to unnecessary treatment of normal women in the single-visit approach. VIA results are operator-dependent, hence subjective, with high interobserver variations, requiring proper quality control and training-assessment protocols, as well as frequent retraining.

There are no uniform criteria for reporting VIA positivity, although there have been good efforts by IARC to objectivise criteria and develop a training manual. Training for VIA can be provided to nurses within about a week. Training consists of a basic course on anatomy, equipment requirements, and visual demonstration of normal and abnormal cervix from slide sets, followed by hands-on training.

Permanent records of VIA testing results cannot be kept, in contrast to Pap slides that can be stored, or printouts of HPV-reading equipments.

VIA is not suitable for postmenopausal women where the squamocolumnar junction is not easily visible any longer and has receded into the endocervical canal.

Evidence shows that providing a single VIA test followed by cryotherapy treatment for test positive cases, can reduce the lifetime risk of cervical cancer by $30 \%$, in women between the ages of 35 and 45 years. Cryotherapy treatment is less suitable for women with high-grade lesions in whom the cure rates range between $71 \%$ and $96 \%$ [15]. Other situations not suitable for cryotherapy include large acetowhite areas, extension into endocervical canal, lesion in vaginal fornix, squamocolumnar junction not visualized, polyps, ulcers, and distorted or atrophied cervix. If there is suspicion of invasive cancer, biopsy and further evaluation is preferable to cryotherapy.

Also side effects and complications are inherent to cryotherapy: $4 \%$ women will have minor side effects (e.g., vaginal discharge, cervicitis, bleeding, abdominal cramps), and $0.5 \%$ will have major complications (e.g., blood transfusion, pelvic inflammatory disease). After cryotherapy, women must abstain from sexual intercourse for a month, or at least use condoms. Doing so might prove difficult in some communities where women are often disempowered and men may be reluctant to comply.

HPV testing for detection of high risk HPV in cervical cells can increase the sensitivity for underlying lesions, though with a decrease in specificity (as it may pick up infection prior to development of lesions) as compared to cytology. More and more data support using primary HPV DNA testing combined with cytology, VIA, or other screening method for triage of women screened HPV-positive. The role of HPV testing as an adjunct to cytology-based screening and as a primary screening test is discussed in FIGO guidance, Section 11 [2]. There are several diagnostic tests for detection of oncogenic genotypes of HPV; some detect HPV-DNA, and others target HPV-RNA. Recent research indicates that HPV testing is the most sensitive screening tool available at this time for the detection of underlying CIN 3 and cervical cancer. In 2009, a randomized controlled trial of over 130,000 women in India showed that a single round of HPV DNA testing by the Hybrid Capture 2 method reduced cervical cancer deaths by about $50 \%$ within the eight years of followup [16]. The HPV test is highly sensitive, although less specific, in primary screening of precancerous lesions of the cervix (CIN 2 and CIN 3). Globally there is high prevalence of HPV in young women, declining in older women, as most have cleared HPV infection by their early 30s. Therefore, focusing HPV testing on women over the age of 30 years is likely to yield the best results.

HPV testing does not have a role for triaging women with clear cytological abnormalities (LSIL or HSIL), since a considerable percentage of these women are HPV infected; adding HPV testing would only add additional cost. But there is significant benefit in using HPV testing to triage women with undetermined cytological changes (ASCUS). The combination of HPV testing and cytology has demonstrated a slight increase in sensitivity for detection of CIN $2 / 3$ compared to HPV testing alone, but this benefit will vanish in areas where cervical cytology performance is suboptimal. HPV screening has also been proposed when VIA is used as a triage tool for women with a positive HPV result.

Currently, one of the factors of HPV testing is the high cost. However, an affordable, rapid, and simple test has been developed and is undergoing field trials [17]. Thus, even in low-resource settings, it is expected that HPV testing can be considered in the very near future.

4.2. Diagnosis. The screening test is only the first step of a successful screening program. The followup of women with positive results, monitoring women with abnormal or undefined results, colposcopy and taking biopsies for histopathology, and treatment of abnormal lesions are necessary additional steps before any impact of the screening program on disease burden can be achieved. The cost of all these components may be prohibitive in some settings. In addition, screening has challenges such as the complexity of coordinating the several steps of the process, engaging qualified health workers, and providing quality control tools.

In the case of cytology screening, colposcopy is essential in making a final diagnosis on the severity of lesions before deciding on treatment. Although colposcopy is not a sufficient tool for screening, as alone it has low sensitivity and low positive predictive value, it is essential in a cervical cytology screening program, to guide the clinician to the area most abnormal for biopsy for histology in order to make a diagnosis of preinvasive or invasive cervical neoplasia.

In screening programs in high-resource settings, colposcopy remains the reference for making the diagnosis, as histological assessment of biopsies taken from acetowhite lesions or other abnormal areas is needed to make a definitive diagnosis following abnormal cytology or any other abnormal test. The indication for colposcopy varies depending on the screening methods used (Refer to the FIGO guidance for more details [2]). Colposcopy is usually carried out in an outpatient setting. It has many fallacies: requires training 
and quality assurance and hence accreditation systems are recommended and common in many countries.

4.3. Preventive Treatment Modalities. High-grade cervical intraepithelial neoplasia lesions (CIN 2+) need to be treated by excision to prevent potential progression to cancer. Although ablative methods such as cryotherapy could be a treatment choice for small, completely visualised lesions, excisional methods such as loop electrosurgical excision procedure (LEEP) or conization allow histological diagnosis and assessment of margins to ensure complete removal especially in larger lesions. The advantages and disadvantages of various methods, special considerations and recommendations, and other issues are further discussed in Sections 13-14 of the FIGO guidance [2]. For instance, there are multiple management options for ablation of cervical dysplasia. Cryotherapy has been in use for over 40 years as a safe and effective way of destroying (ablating) CIN lesions on the ectocervix, by freezing the cervical epithelial tissue. Cells rapidly reduced to -20 degrees Celsius for three to five minutes will undergo cryonecrosis. Cryotherapy is well suited for low-resource settings. A review of the literature shows a cure rate of $90 \%$ at one-year, and over $85 \%$ of women found the procedure to be safe and highly acceptable [18]. Cervical cone techniques currently used include cold knife conization (CKC), loop electrosurgical excision procedures (LEEP/LLETZ), and laser conization. All three techniques are effective in the treatment of CIN and studies have found no difference in the sample adequacy between the techniques [19]. No matter what the choice of treatment is, accessibility and quality assurance should be ensured, as well as ongoing monitoring and supervision of provider skills.

\section{Treatment of Invasive Cancer or Tertiary Prevention}

For those women where screening detects invasive cervical cancer, available standard-of-care treatment should be offered in order to achieve the goal of controlling the spread of cervical cancer. Depending on the stage of invasive cancer, the treatment choice may be surgery, usually radical hysterectomy, with pelvic lymphadenectomy, radiotherapy, or concurrent chemoradiation. Treatment of invasive cervical cancer requires multidisciplinary contributions from gynaecological oncologists, radiation oncologists, medical oncologists, radiologists, and nurse specialists. Establishment of a regional treatment centre with appropriately trained specialists could be a challenge in low-resource settings, and in rural areas. FIGO guidance, Section 17 [2], provides information on advancements in this area and recommendations regarding optimal therapy choices.

\section{Special Considerations in Low-Resource Settings}

6.1. Primary Prevention. The vast majority of AOGIN member countries or regions consider that HPV vaccination to teenagers before sexual exposure should be implemented at large, in accordance with the WHO position and recommendations.

The obstacle to low-resource countries is mainly the lack of national support in terms of financial resources and infrastructure to ensure large coverage adolescent vaccination programs are instituted. Among the various vaccination programs implemented so far, the strategy of delivering HPV vaccines to school girls, 11-13 years, has shown good coverage rates $[20,21]$. Combining HPV vaccination with delivery of other health interventions or education to adolescent girls, such as vitamin A, iron supply or HIV preventive education, or tetanus toxoid immunisation (TT) or meningitis vaccination may increase the cost-benefit of such programs. Public awareness and health workers' education is needed for compliance and full implementation of effective vaccination. The way forward for health professionals is to educate the women, maintain an open dialogue with local health authorities, and inform them about the benefits of preventive interventions, while continuing to advocate for financial support from national health ministries, as well as to seek support from international procurement and health agencies such as GAVI, UNICEF, WHO, and The Global Fund.

6.2. Secondary Prevention. The vast majority of AOGIN member countries consider that cervical cancer screening should be offered to target populations, namely sexually active women, particularly over 30 years of age. The choice of different screening methodologies and strategies, for example, VIA, VILI, rapid affordable HPV test, or cytology, should be carefully considered based on accurate assessments of the sensitivity, specificity, diagnostic values, time to deliver results, coverage, follow-up rates, and cost-effectiveness analyses.

When planning screening programs, six key elements should be considered in order to ensure success, as listed in Table 1. The choice of the screening method is the first step, and should be based on local data of sensitivity and specificity of each method, as these can vary depending on the prevalence of HPV in the population.

For low-resource settings, the simplest method is VIA/VILI or, in the future, HPV detection using a low-cost test. It is desirable to use an adjunct method to confirm screening results using cytology, colposcopy, or cervicography and biopsy, before referring women to treatment, although "screen and treat" should be considered, depending on disease prevalence, infrastructure for delivery of services, rates of compliance to followup, and resources. At least cryotherapy should be available to treat identified high-grade lesions, and surgery to remove invasive cancers. Screening should start at age 30 or 35 years, depending on local data on HPV prevalence and incidence of cervical neoplasia, as well as resources. Screening should be offered to all women at least once in their lifetime, but preferably every 5 years, if using VIA, or every 10 years if using HPV testing. National cancer registries should be in place to be able to measure disease burden and the advancement and success of prevention programs. 
TABLE 1: Key elements to be considered in choosing a screening strategy for low-resources settings.

\begin{tabular}{ll}
\hline Screening method & $\begin{array}{l}\text { VIA/VILI or HPV DNA detection (low } \\
\text { cost) }\end{array}$ \\
\hline Adjunct & $\begin{array}{l}\text { Cytology or cervicography or colposcopy } \\
\text { with biopsy } \\
\text { Treatment for CIN 2+ for example } \\
\text { cryotherapy, cold coagulation; and } \\
\text { treatment for cancer such as surgery and } \\
\text { radiotherapy }\end{array}$ \\
Support & $\begin{array}{l}\text { Starting at 30-35 years } \\
\text { Once in a lifetime or every 5-10 years }\end{array}$ \\
Age & National level coordination \\
\hline
\end{tabular}

Importantly, at least once or twice in a lifetime screening is strongly recommended to be considered based on individual countries' preferences and availability of resources. Similar to vaccination, public awareness and health workers' education is needed for full implementation, to ensure compliance and a successful program.

\section{Special Considerations in High-Resource Settings}

7.1. Primary Prevention. The aim is to provide HPV vaccination to target populations. Among AOGIN member countries, Australia and New Zealand so far were the first to offer HPV vaccination to target populations ( 12 year old, and in the first two years of the program catch up to 26 years old) supported by the government [22]. Recently, the Malaysian government has intended for vaccination and will be offering a government-funded program. Though both HPV vaccines have been licensed and registered for use in other AOGIN member countries, they are currently administered based on self-financing and in an opportunistic manner.

The way forward is to continue to inform governmental authorities about the disease burden and potential benefits and to advocate for political support to adopt HPV vaccination, as part of national immunization programs. In addition, education of health workers and of the public at large is needed for full implementation and high compliance.

The modalities of pricing and reimbursement depend on local health systems and local legislation and policies, as well as negotiations between governments and health insurers. Some countries have also created reimbursement mechanisms such as those based on centralized government procurement and distribution for negotiated lower prices, but administered by private practitioners. Other countries provide reimbursement of the vaccination course, after purchase by the patients and proof of administration of the three required doses by a practitioner.

7.2. Secondary Prevention. The aim is to provide cervical cancer screening to target populations.

Many AOGIN member countries have been using cervical cytology screening with a variety of programs and,
TABLe 2: Key elements to be considered in choosing a screening strategy for high-resources settings.

\begin{tabular}{|c|c|}
\hline Screening method & Cervical cytology or HPV DNA testing \\
\hline Adjunct & $\begin{array}{l}\text { HPV testing and colposcopy for } \\
\text { abnormal cytology, colposcopy, and/or } \\
\text { biopsy for those persistently HPV } \\
\text { DNA }(+)\end{array}$ \\
\hline Support & $\begin{array}{l}\text { Colposcopy and treatment for CIN2+ } \\
\text { and cancer }\end{array}$ \\
\hline Age & $\begin{array}{l}\text { Starting at } 25 \text { to } 30 \text { years old and ending } \\
\text { at } 65 \text { years old }\end{array}$ \\
\hline Interval & Once every 3 to 5 years \\
\hline Screening Registry & Available \\
\hline Cancer Registry & $\begin{array}{l}\text { National, regional, and local level } \\
\text { available }\end{array}$ \\
\hline
\end{tabular}

indeed, decrease in incidence and mortality from cervical cancer was demonstrated, for example, in Australia and in Taiwan.

While the provision of screening is essential to allow reduction in disease burden, having registries for cytology and cancer are important elements to help in monitoring the success of programs. Furthermore, financial and government support is essential for a successful population-based screening program.

In the United States for example, cytology screening with HPV testing as an adjunct is recommended for women over 30 years of age, and at intervals of three years upon negative results. Screening using high-risk HPV testing shows higher sensitivity, though lower specificity, and this method is being explored as a primary screening tool in a number of research settings. Algorithms in using HPV testing with or without cytology are yet to be agreed upon in Asian countries. The use of cytology screening followed by HPV testing, or HPV testing followed by cytology for confirmation, is likely to be used, rather than VIA or VILI. For definitive diagnosis of lesions, colposcopy and histopathology should be encouraged. Treatment modalities in high-resource settings should be based on LEEP/LLETZ or conization, rather than on cryotherapy. Surgical and radiotherapy treatments are available in most high-resource settings. Cancer registries have shown their utility in monitoring disease burden and success of prevention programs. In some high-resource settings, screening registries are also available and allow call-recall mechanisms and follow-up procedures, facilitating quality assurance schemes and providing good quality data and corrective actions to improve the effectiveness of the programs. The key elements to be considered in choosing a screening strategy for high-resources settings are summarized in Table 2.

Public awareness and health workers' education and training will be essential, especially if HPV testing is to be used for screening. The potential stigma associated with genital HPV infection could lead to resentment and guilt feelings among those who screen positive for HPV. 


\section{Special Considerations and Recommendations}

8.1. AOGIN Recommendations for Screening Programs in LowResource Settings. Affordable, effective strategies have been proposed that could work in low-resource settings.

8.1.1. Visual Inspection Techniques. These include visual inspection with acetic acid (VIA) and visual inspection with Lugol's iodine (VILI). These tests are suitable for women of the reproductive age group 30-50 years, but not after the menopause, since the squamous junction recedes into the ectocervical canal and the entire transformation zone is no longer visualised (Figure 2). The advantages of VIA/VILI are

(i) simple, inexpensive, minimally reliant upon infrastructure,

(ii) easy to learn, can be performed by many levels of health care providers (including nurses and midwives) after short training, may be integrated into primary health centre services, and

(iii) results are available immediately, thus can be integrated into screen and treat strategy.

The sensitivity of VIA ranges between $66 \%$ and $96 \%$, with specificity between $64 \%$ and $98 \%$ [23]. Low-level magnification (VIAM) did not improve the test performance [24]. Addition of VILI has been shown to improve the sensitivity and specificity of VIA [25]. Health workers find the colour gradients of VILI easier to define than VIA. Detection rates of CIN 2/3 using VIA, VILI, and cytologic findings of ASCUS and LSIL were 3.7, 3.3, 4.5, and 4.2 per 1000 women, respectively [26].

8.1.2. HPV Testing as "Single Round of Screening". In a low-resource setting, it is difficult to ensure screening at regular intervals. Sankaranarayanan and collaborators have shown that in a low-resource setting in rural India, a single round of high-risk HPV DNA testing by Hybrid Capture 2 technique reduced the rate of advanced cervical cancers and associated deaths by $50 \%$, as compared with the unscreened control group over 8 years followup. Also, a single round of cytologic-testing or VIA in a similar setting was not associated with such a significant reduction in advanced cervical cancer and related death rates. The age-standardized rate of invasive cancer among screen-negative women was almost four times lower in HPV-negative women compared to cytology-negative and VIA-negative women, indicating that a single negative HPV DNA test had a higher negative predictive value than the other methods [16].

HPV testing is sensitive, objective, reproducible, and can even be performed by a technician. Presently the drawback is that HPV testing is expensive. A rapid affordable test (careHPV ${ }^{\mathrm{TM}}$, Qiagen Inc.) is expected to become available by 2011-2 [17]. Thus, implementation of a nationwide program of once in a lifetime testing around age 40 years with lowcost HPV testing holds significant promise for rapidly and substantially reducing the cervical cancer burden (Figure 3 ).
8.1.3. Screen and Treat Modalities. The single visit approach ensures high compliance and reduces wastage of limited resources in screening women who will not return for management of an abnormal screening test. The best age for a once in a lifetime screening is between 35 and 40 years.

Visual inspection and treatment by cryotherapy at the same visit for ectocervical lesions that can be covered by a cryoprobe was found to be safe, acceptable, and feasible [27]. This screen-and-treat method effectively cured CIN in $88 \%$ women, including $70 \%$ women with a baseline diagnosis of CIN 3 [28]. A randomized trial in Southern India found a $25 \%$ reduction in cervical-cancer incidence and a 35\% reduction in mortality compared to controls, with VIA followed by cryotherapy [29]. If facilities permit, a biopsy should be taken prior to cryotherapy for post hoc confirmation of diagnosis, if possible. Loop electrosurgical excision (LEEP) is recommended for larger lesions.

Screen-and-treat strategies have also been tried with cytology [30] with a rapid turnaround time for the reporting of cytologic results and HPV testing [31]. These overcome the problem of loss to followup but still present the problem of infrastructure and cost.

\subsection{AOGIN Recommendations for Screening Programs in}

High-Resources Settings

8.2.1. HPV Testing. Persistent infection with high-risk HPV types is a necessary cause of cervical cancer and high-risk (hr) HPV testing as a screening test has 20-40\% greater sensitivity, but 5-10\% lower specificity than a Pap smear [32]. Women who were hrHPV DNA negative appear to be at low risk for CIN $3+$ for up to 10 years (high negative predictive value (NPV)) [33]. Liquid cytology with automated reading may be also highly objective and reproducible for high-resource settings. The NPV of being both cytologically and hrHPV DNA negative is extremely high, over $99.9 \%$ in most studies.

Switching from a cytology-based to an HPV-based strategy will detect 120-300 extra cases of CIN 2+/100,000 women screened. Thus, a strong case should be made for considering utilizing HPV DNA testing as the primary screening test in women aged over 30 years [34] (Figure 4). Five to $8 \%$ of women over 30 years of age will be high-risk HPV positive. Cytology may be used to triage HPV-positive women into 2 groups: those requiring colposcopy and those who can be followedup (Figure 5). The higher specificity of cytology makes it ideally suited to rule out high-grade lesions/invasive cancer in HPV positive women.

HPV DNA-positive, cytology-negative women should be retested in 12 months with both cytology \& HPV DNA testing: there is no immediate necessity for colposcopy. Persistent HPV infection does require colposcopy. Fourquadrant biopsies, plus endocervical curettage, has been recommended even if there is no obvious lesion on cytology in these cases [35].

For women who are both cytology and HPV negative, the screening interval may be safely increased to 8 or 10 years [36-38]. 


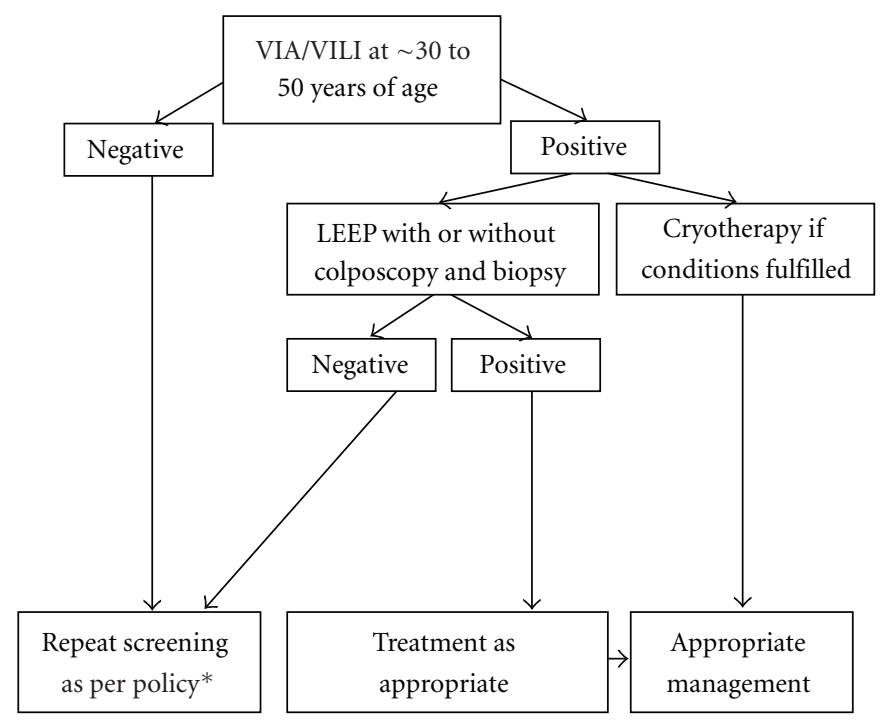

* Once or twice in a lifetime with 10 years interval or

every ten years or

every five years or

every three years

FIGURE 2: Schema for low-resource situations starting with VIA-based screening.

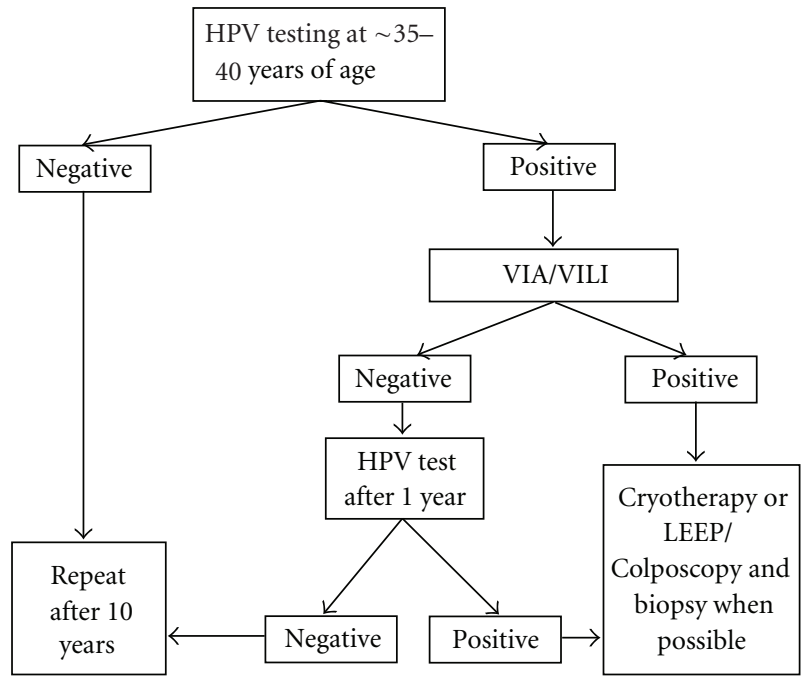

FIGURE 3: Schema for low-resource situations, starting with affordable HPV testing.

Some of the strategies that can be incorporated in an HPV-based screening program in high resource settings include the following.

(a) "Reflex" cytology: A specimen is obtained from all women using a liquid collection media suitable for both HPV DNA testing and cytology. Specimen is initially tested for high-risk HPV, but processed for cytology only if HPV positive. This approach would eliminate the need for cytology in $90 \%$ to $95 \%$ of women, and will depend on the availability or not of cytotechnicians in the setting.

(b) HPV genotyping: Women with persistently positive specific high-risk types of HPV (types 16, 18, 33, 45, or 31) are at higher risk for having underlying or subsequent CIN2+ (referred for colposcopy), while those with other hrHPV types are at a lesser risk (repeat HPV test in 12 months) ultimately for cancer cervix. Commercially available HPV genotyping assays to identify the specific type of HPV are now being considered for introduction for clinical use [39]. Whilst there are some guidelines for testing with high-risk HPV DNA assays, there are no formal guidelines yet for genotype-specific assays.

(c) "Reflex" cytology + HPV genotyping: The third option is to screen using hrHPV testing and then use a combination of both reflex cytology and HPV genotyping to identify which HPV DNA-positive women need colposcopy. This may be justified only in settings where access to colposcopy is limited and high cost. Cost-effectiveness analysis may be conducted before establishing the strategy.

8.2.2. Cytology as Primary Screening. This is recommended for screening women over 30 , and also in women under 30 years who have been sexually active for over 3 years. Liquidbased cytology has some advantage of improved sensitivity and fewer unsatisfactory results compared to conventional cytology by Pap. 


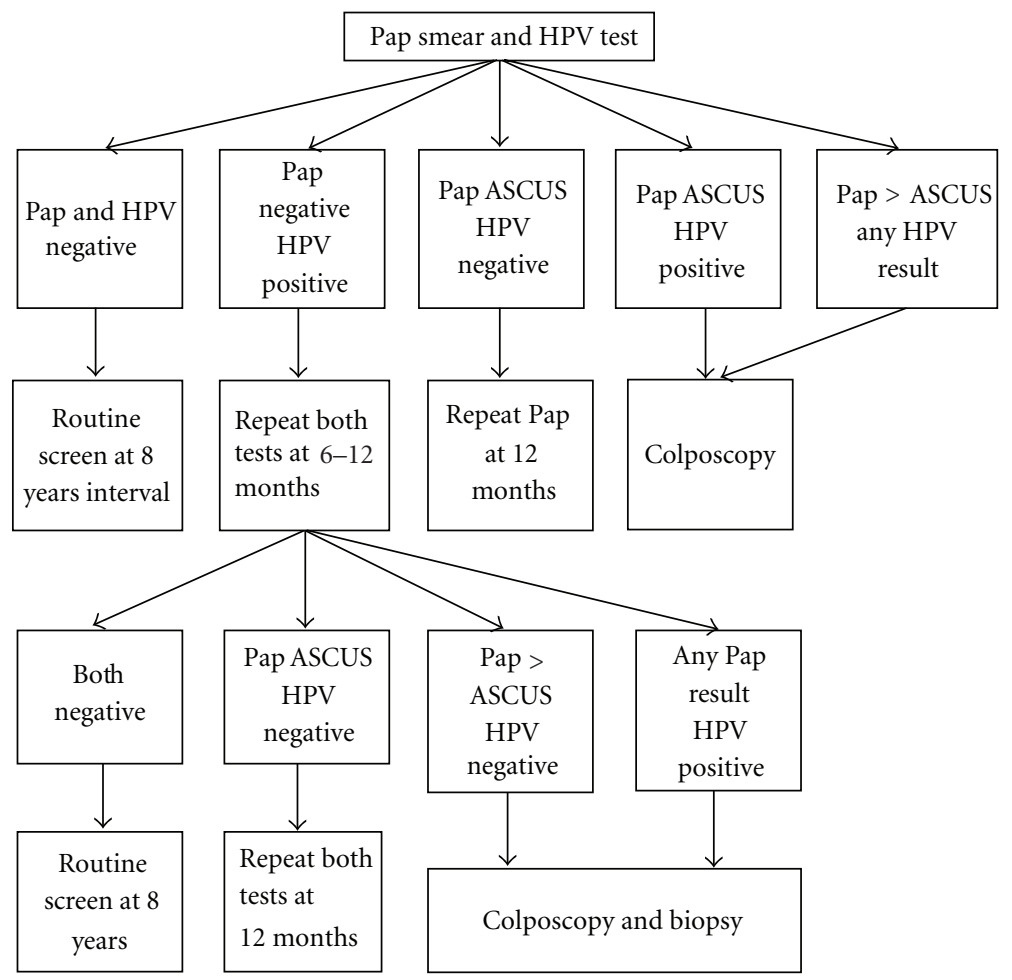

FIGURE 4: Schema for high-resource situations, starting with parallel testing with cytology \& HPV DNA testing.

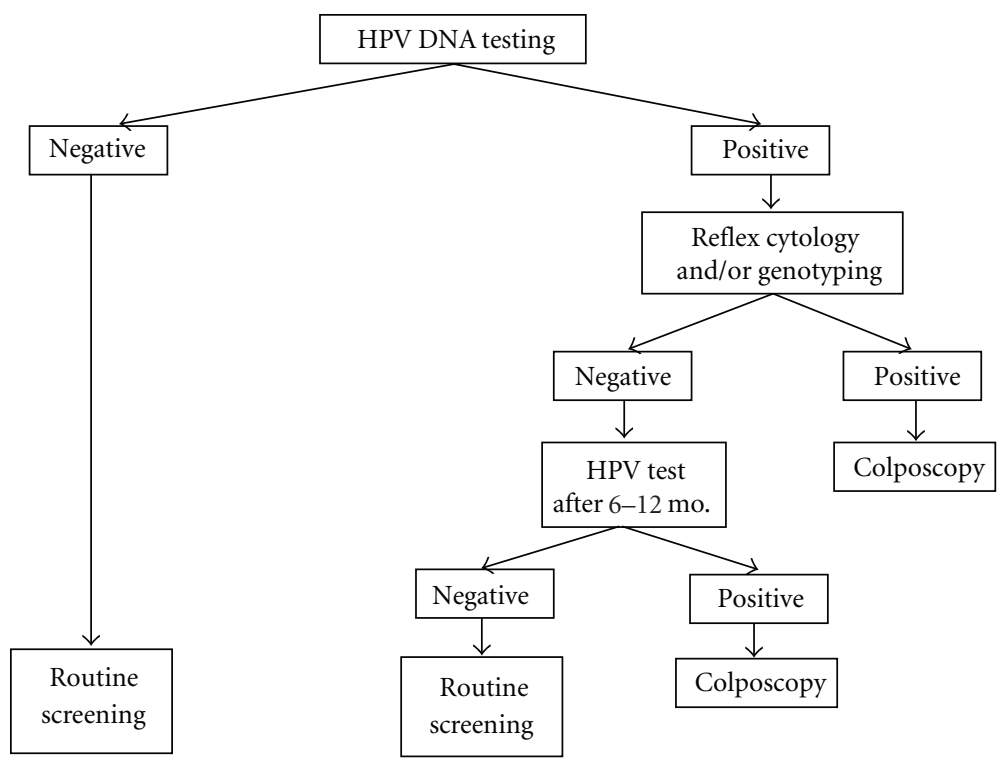

FIGURE 5: Schema for high-resource situations, starting with HPV DNA testing with reflex cytology and/or HPV genotyping.

\subsection{Special Considerations for HPV Vaccination Programs}

8.3.1. HPV Vaccine Efficacy and Safety. The HPV L1 VLP vaccines are safe and well tolerated. They are highly immunogenic. These vaccines are essentially empty protein shells that consist of virus-like particles (VLP) and do not contain viral DNA; so these vaccines are not infectious and nononcogenic. Phase 3 clinical trials have documented the safety, efficacy, and immunogenicity of the quadrivalent and bivalent HPV
L1 virus-like particle vaccines in preventing vaccine-related HPV high-grade dysplasia in those initially naive to HPV 16 and/or 18. These data have been presented to regulatory authorities in over 100 countries around the world who have provided approval for marketing of the products for use in target populations. High sustained efficacy of the prophylactic vaccines up to 5 and 8.5 years of followup have been reported [10, 40, 41]. 
The bivalent HPV vaccine protects against the two major cancer causing HPV viral types 16 and 18, and the quadrivalent HPV vaccine protects against four viral types $6,11,16$, and 18, thus making it effective for prevention of cervical cancer, as well as for vulvar and vaginal warts. Both these vaccines translate into protection of cervical cancer in the order of 70-75\%, which represents the percentage of invasive cancers attributable to HPV-16 and -18 . Both vaccines have demonstrated greater than 93\% efficacy in preventing HPV-related high-grade cervical neoplasia (CIN2+). In addition, the quadrivalent vaccine has been shown to be $100 \%$ efficacious in preventing vulvar intraepithelial neoplasia (VIN) and vaginal intraepithelial neoplasia (VanIN2+) [42], and showed $100 \%$ efficacy in preventing genital warts.

Noteworthy, they also showed efficacy against CIN2+ caused by other oncogenic HPV types, phylogenetically related to HPVs 16 and 18. For instance, the bivalent and the quadrivalent vaccines showed, respectively, $68 \%$ and $33 \%$ efficacy against lesions specific to HPV types 31/33/35/39/45/51/52/56/58/59 [43].

Adverse events associated with the HPV vaccines are much the same as with other vaccines, such as hepatitis $B$ vaccines, with respect to fatigue, headache, myalgia, gastrointestinal complaints, arthralgia, elevated temperature, rash, or urticaria. Fainting after HPV vaccination was found to be more common among teens than among young children or adults [44]. However, fainting among teens is most often a response to the injection process rather than a side effect of the vaccine. A study in the United States showed that fainting was not more common after HPV vaccination compared to other vaccines given to teenagers and young women. Therefore, as with other vaccines, a standard fifteenminute resting period is recommended postvaccination to prevent any injury associated with fainting [45].

Events involving hospitalization, death, disability, lifethreatening illness, or other medically important conditions account for approximately 3 per 100,000 events reported to the US Vaccine Adverse Events Report System (VAERS) in the US. There does not appear to be any significant difference in the number or severity of adverse events between the two HPV vaccines [46].

Other potential vaccine-related adverse effects include Guillain Barre Syndrome (GBS), venous thromboembolic events (VTEs), and serious allergic reactions. However, when reviewing the background rate of these events within the age-matched general population, HPV vaccines have not been shown to increase risk of these events among women with any known risk factors. The relative risk of venous thromboembolic events (VTEs) such as deep vein blood clots is 2 per 1,000,000 individuals vaccinated with Gardasil in the US. Ninety percent of cases (28 of 31 VAERS reports, all in women ages 15-39) had a known risk factor for VTEs including use of oral contraceptives and family history [47].

8.3.2. Recommended Immunisation in Low-Resource Settings. The ideal age for the administration of the HPV vaccines is between 10 and 13 years, and an effective way to deliver them while maximizing compliance to the three doses is in schoolbased vaccination campaigns or programs, where delivery is also likely to be cost-effective. As previously mentioned, a standard fifteen-minute resting period is recommended for adolescent postvaccination to prevent any injury associated with fainting. Moreover, ensuring that the individual girl who has been vaccinated is not within view of others about to be vaccinated is helpful. The vaccines are available in a single-dose $(0.5 \mathrm{~mL})$ vial or prefilled syringe; no dilution or reconstitution is required. The vaccine is administered intramuscularly as three $0.5-\mathrm{mL}$ doses, injected into the deltoid region of the upper arm. Vaccination of males in the context of large scale public vaccination programs or campaigns is not recommended, as the potential costeffectiveness, cost-benefits, and herd immunity effects have not yet been fully demonstrated. However, in considering equity and prevention of other HPV-related diseases in males, as well as herd immunity, should uptake in women be reduced, vaccination of males may well be appropriate.

\subsubsection{Recommended Immunisation in High-Resource Settings.} In Western Europe, for example, where healthcare budgets and existing infrastructure can more easily accommodate the introduction of new vaccines, nine countries (Denmark, Germany, Greece, Italy, Luxembourg, Netherlands, Portugal, Spain, and the UK) offer HPV vaccination free of charge to at least one age-cohort of females, and additional 3 countries (Belgium, France, and Sweden) offer HPV vaccination on a copayment basis. Australia is the first country to implement a comprehensive school-based governmentfunded schoolgirl vaccination, while in western Europe, the UK and Switzerland have implemented a nationwide school based vaccination program, while in Spain only 9 of the 19 autonomous regions currently deliver HPV vaccines through this mechanism. School-based vaccination programs are known to achieve high coverage of the target population for paediatric and adolescent vaccines [48] while on-demand provision through healthcare providers, even if supported by direct invitation and/or public education programs, usually has suboptimal coverage rates and can lead to missing lower socioeconomic groups and minorities.

In high-resource settings, HPV vaccination of girls is recommended for girls around 12 years old, and in addition catch up vaccination for girls up to 15-18 or even extended up to 26 years of age, depending on the country resources and decisions, as extended catch up could accelerate the impact of vaccination on reduction of cervical cancer incidence [49].

8.3.4. Vaccination of Mid-Adult Women. While the priority of vaccination programs is to target young girls and women, benefits of vaccination continue even in older women as there is a continuing risk of new HPV infections throughout life, and infections acquired in later life are more likely to be persistent. For sexually active women over the age of 25 years, vaccination can be considered on an individual basis as most will have the potential to benefit from vaccination. Evidence from clinical trials suggests benefit to women aged $18-25$ years similar to the $15-17$ years age group. There is 
no recommendation for HPV testing as a prerequisite to vaccination as it is unlikely that women would be positive for all HPV types included in vaccines.

8.3.5. Postmarketing Surveillance. Surveillance needs to be sensitive enough to document any unexpected adverse events that occur after immunization, but also recognize that in any population over a given period of time, such as a year, new cases of diseases and health problems may arise in its members. Whilst both the detection of adverse events following immunization, and the subsequent requirement to disentangle chance association from causality, are challenges faced by any vaccination surveillance system, HPV vaccination programs potentially face a specific challenge in relation to vaccine safety surveillance. This challenge relates to the target group for vaccination comprising preadolescent and adolescent girls and young women. Incident diseases in this population group are different from those that occur with the traditional infectious diseases framework of children, and who are the usual target group for large scale vaccination programs. Moreover, the time frame in which the disease (cervical cancer) occurs is so much greater than traditional infectious diseases.

Consequently, HPV vaccination surveillance systems will need to be able to refer to background rates of diseases in the target population to determine whether cases of new onset disease reported after vaccination are occurring at a greater than expected rate and, just as importantly, be able to communicate about such occurrences and rapidly investigate through data linkage studies if necessary. Not all countries may be able to achieve this; it will require registries for HPV vaccination and cervical cytology, as well as cancer registries, and the ability to link them to changes in disease outcome.

As far as possible recording of routine adverse events following immunization (AEFI) notifications and vaccine safety assessments should be standardized in order to improve accuracy and completeness of information about adverse events both in pre- and postlicensure clinical studies as well as in safety surveillance systems [50]. A summary of reports to the vaccine adverse event reporting system (VAERS) following receipt of quadrivalent HPV concluded that most of the AEFI rates were not greater than the background rates compared with other vaccines, but there was disproportionate reporting of syncope and venous thromboembolic events. The significance of these findings must be tempered with the limitations of a passive reporting system. In addition, an analysis of the HPV-16/18 bivalent vaccine trials alone was also undertaken $(N=39,160)$ and demonstrated a low rate of autoimmune disorders, without evidence of an increase in relative risk associated with AS04 adjuvanted vaccines [51].

Based on the official statements of national and international agencies and expert immunisation groups (WHO, CDC, FDA, PHAC, ATAGI, EMEA, STIKO, PEI, AFSSAPS, and others) to date, as well as a series of reports, HPV vaccines seem to be safe and effective vaccines of great importance for women's health. However, it is vital that continued and careful monitoring is undertaken so that any rare but potentially important adverse events can be detected, and verified, if and when they occur.

8.3.6. HPV Vaccines and Pricing. To date, the price of HPV vaccines varies from country to country and dependent on whether purchased in the context of private or public vaccination. The available information on pricing in various countries varies from over 200 US dollars per dose in the private sector in Switzerland, 150 US dollars per dose in Hong-Kong, 120 US dollars per dose in Australia and Singapore, down to 30 US dollars per dose, in public programs in the Philippines and reduced now to $40 \mathrm{US}$ dollars in India for example. Public nationwide programs are likely to negotiate lower prices, than in the private sector vaccination, and drive prices further down. In addition, for resource-poor countries, the manufacturing pharmaceutical companies have agreed to cost down price.

8.3.7. Screening after Vaccination. Screening has to continue after vaccination as up to $30 \%$ of cervical cancers may not be prevented by currently available vaccines, women may have acquired infection prior to vaccination and there may be some cases in spite of vaccination. At the present time, there is no change in the screening recommendations, but it is expected that with time there will be fewer cytological abnormalities, which will lead to further lowering in the accuracy of cytology and greater emphasis on HPV testing as a screening method.

\section{Contributions from Some Countries within the AOGIN Region}

It is important to share our experiences within the Asia Oceania region, in order to facilitate the formulation of national guidelines that suit the purpose of each country. In the following section are contributions from AOGIN member countries or regions sharing their experience on cervical cancer control, namely from Australia, China, HongKong, India, Korea, The Philippines, Singapore, Taiwan, and Thailand.

\subsection{Australia}

\subsubsection{Current Situation in Cervical Cancer Control and Prevention}

(a) Primary Prevention: Prophylactic Vaccination. Australia registered the quadrivalent vaccine in June 2006, the only one registered at that time. In November that year, the Australian government announced funding for an HPV vaccination program which was initiated in April 2007, as a school-based, ongoing program for 11- to 13-year-old girls [22].

In addition, for the first two years of the program, a one-time funded catch-up vaccination opportunity phase for those up to 26 years of age, began in July 2007 and ended in December 2009. It was largely delivered in school-based programs, and for women aged 18 to 26 years, through general practice (GP) and community-based immunisation 
providers. The ongoing program for 11-13-year-olds is now in its fourth year and is an ongoing, annual program delivered in the first year of high school [22]. By the end of 2008 , the bivalent vaccine had also been approved for use in this program.

Australia is a world leader in the success of this HPV vaccine program, as the well-organized program high uptake, of nearly $80 \%$, and with around $75-80 \%$ estimated uptake in the catch-up program. By May 2009, over 5 million doses of the quadrivalent vaccine had already been distributed in Australia. The program's effectiveness has already translated into a reduction in genital warts, the first indicator of success of the program in a real world setting. In a retrospective review of new cases of genital warts being seen in the Melbourne Sexual Health Clinic, since the introduction of the national program for young women, irrespective of their vaccination status, the proportion of women under 28 years with warts diagnosed decreased by almost $50 \%$ (48\% in 2008) but not in women over 28 years of age. Of note, since 2008 there has been a significant decline in genital warts in heterosexual men $(17 \%)$, who did not receive vaccination, suggesting a herd immunity effect of HPV vaccination [12].

The next expected change that would be anticipated would be in rates of abnormal Pap smears and we look forward to review of this through the HPV vaccine register, which is also part of the HPV vaccine program. This will ultimately allow for measures of vaccine effectiveness in reducing cervical cancer burden on a population basis, by linkages of well-established cancer, Pap cytology, as well as vaccine status.

(b) Secondary Prevention: Cervical Cytology Screening. Australia has a very effective secondary prevention program for cervical cancer, the National Cervical Screening Program (NCSP). The program provides organized, cervical cytology screening with high coverage, as well as high quality laboratory assurance, in addition to adequate treatment for precancerous lesions. The NCSP currently consists of regular ( 2 yearly) Pap testing, targeting women aged 18 (or 2 years after sexual debut) to 69 years. The NCSP program is well organized and commenced comprehensively in 1991. The success of this is exemplified by the significant drop in incidence of cervical cancer in women of all ages decreasing from 12.7 to 4.9 per 100,000 , with mortality decreasing to $1.4 / 100,000$, one of the lowest worldwide [52]. Hence cervical cancer has now fallen to the eighteenth most common cause of cancer death among Australian women in general. Notwithstanding, the success of the NCSP, however, are the higher incidence and mortality rates for cervical cancer in indigenous women. Incidence and mortality rates in indigenous Australian women approach those close to developing countries with mortality rates in 2001 being over four times greater than nonindigenous women, and incidence rates 4 to 5 -fold higher. This is largely because of lack of ready access to services and resultant poor screening services particularly in remote areas [53].

\subsubsection{Challenges and Suggestions to Overcome Obstacles}

(a) Vaccination of Males. Clinical phase 3 trial data in males of 16-26 years for the quadrivalent vaccine show it is well tolerated, induces a strong type-specific immunological response comparable to that of females, and reduced vaccine HPV-type-related genital infection, as well as disease, including genital warts as well as anal intraepithelial neoplasia due to vaccine related HPV types $[54,55]$.

Ultimately, comprehensive cost-benefit analyses are needed to determine the efficacy of male as well as female programs in the overall Australian population [56].

(b) Innovative Screening Strategies. With an increased vaccinated population of young women, the positive predictive value (PPV) of Pap cytology will decrease. Hence the current program is shortly to be reviewed and may result in changes to the age of commencing screening as well as use of a more sensitive assay such as HPV DNA and use of cytology for triage. This will be reviewed as part of the Government Renew Program shortly to commence.

(c) Education. Appropriate communication to the general public about cervical cancer vaccines will vary between cultures and countries. It was noted, for example, in an Australian survey, that endorsement by the medical profession, and professional bodies influenced individual's choice on adoption of a vaccine program [57-59]. This was found similarly for women in Singapore, and despite the general lack of knowledge regarding HPV and the vaccine availability among the general public [60]. Of note, Singaporean men were found to have moderate knowledge of cervical cancer but poor knowledge and awareness of HPV. Although these men showed strong support for HPV vaccination, overall findings highlight the importance of including men in education campaigns that aim to decrease the incidence of cervical and other HPV-related cancers and to increase the uptake of HPV vaccination [61].

\subsection{China}

9.2.1. Current Situation in Cervical Cancer Control and Prevention in Main China. In China, there is not yet a nationwide program for cervical cancer prevention. Neither of the HPV vaccines has been licensed yet in China. The priority for cervical cancer control is secondary prevention by using less expensive, easy to operate, and appropriate screening technologies.

In recent years, China's government has put the health of rural women firmly on their agenda and is focusing its efforts on secondary prevention of cervical cancer. Started in 2006, two demonstration sites for the early detection and treatment of cervical cancer have been established in rural areas, as well as in urban areas for comparison, sponsored by the Ministry of Health and the Cancer Foundation of 
China (CFC). At present, free cervical cancer screening examinations by either Pap smear or VIA/VILI have been available for women between 35 years and 59 years under a government-sponsored program proposed by $\mathrm{MOH}$ and the All-China Women's Federation. It is expected that over the next 3 years, 10 million women from rural areas in 221 counties will be able to access this service. It is a part of the country's ambitious 850 billion RMB (US $\$ 124$ billion) health reform plan. The plan will concentrate on China's less developed central and western regions, and cover broad preventive and screening efforts, ranging from vaccination drives to improved cooking and sanitation facilities in rural areas.

9.2.2. Ideal Situation in Low- and High-Resource Settings. Considering the large differences in the rates of cervical cancer as well as the health and economic situation across geographical areas of China, it would be reasonable to develop ad hoc regionally tailored screening strategies that best address specific needs of the different areas.

It is broadly accepted that well-organized cervical cancer prevention programs based on screening with cervical cytology lead to impressive reductions in cervical cancer rates in developed countries. However, it has not been possible to transfer this expertise and successful experience to the developing world, where the infrastructure for cytologybased screening either does not exist or has been problematic.

While both the cytology test and HPV test are not widely available for women in rural China, visual inspection with $5 \%$ acetic acid (VIA) and visual inspection with Lugol's iodine (VILI) reported acceptable values of sensitivity and specificity for CIN2+ disease detection among Chinese women $[62,63]$. In addition, the costs of VIA/VILI implementation are low.

A newly developed HPV DNA test ( careHPV ${ }^{\mathrm{TM}}$ ) has been shown to be a very promising option for cervical cancer screening in China and other low- and middle- socioeconomic populations. The care $\mathrm{HPV}^{\mathrm{TM}}$ test is specifically designed for screening women in low-resource regions of the world, where diagnostic laboratory facilities are not available, in order to identify individuals at high risk of developing cervical cancer [17]. The test can detect the presence of 14 high-risk or potentially cancer-causing types of HPV. The test also can be useful in other low-resource regions of the world such as India, Africa, and Latin America. The test is able to rapidly produce accurate resultswithin 3 hours - thus eliminating the need for additional patient visits to health clinics for further evaluation and treatment. For instance, a woman found to have a high-risk $\mathrm{HPV}$ infection can undergo further confirmatory diagnostic testing immediately by colposcopy or VIA, and in certain cases, get immediate treatment, if desired.

\subsubsection{Challenges and Suggestions to Overcome Obstacles}

(a) Education. China's large population size (total population of $1,337,411,000$ ), along with its ongoing economic and cultural metamorphosis, creates enormous challenges in health care.

Cervical cancer is over-represented in rural areas-owing to lack of education, poverty, and inability to pay for health care. The ambitious government-sponsored program could be a step towards provision of universal cervical cancer screening nationwide, with an estimated 300 million eligible women in China.

This is the first time that the Chinese government has proposed to gradually widen access to public health services so that women in rural China are included. To achieve high coverage of screening it would be necessary, firstly, to inform the female population through educational campaigns about the disease and prevention programs; secondly, to ensure that screened women would receive the results and confirmatory diagnosis on the same day; thirdly, to validate the referral system and treatment clinics, as well as the respective cancer registries. However, the health service capability and the public health challenges are substantial.

(b) Innovative Screening Strategies. The careHPV ${ }^{\mathrm{TM}}$ test is promising as an affordable screening method for public health cervical cancer prevention programs in low-resource settings. We should develop and strengthen the partnerships between NGOs, the public-sector, the private-sector, and health care professional organizations. It is also imperative that these programs address the needs of the poor and ensure that all members of society benefit equally. Government, civil society, and charities should be responsible for ensuring that this objective is met.

\subsection{Hong Kong}

9.3.1. Current Situation in Cervical Cancer Control and Prevention in Hong Kong. In 2006, cervical cancer was the fifth most common female cancer in Hong Kong. According to the Hong Kong Cancer Registry, there were 459 new cases of cervical cancer, accounting for $4.2 \%$ of all new women cancer cases. The median age at diagnosis was 54 years. Meanwhile, cervical cancer was the eighth most common cause of female cancer registered deaths in 2007. There were 129 female registered deaths due to this cancer, accounting for $2.7 \%$ of female cancer registered deaths.

Both the bivalent and quadrivalent vaccines are available in Hong Kong. There is however no national vaccination program. The vaccines are widely available in most private clinics and women health centres. There is wide media coverage of both vaccines, but uptake statistics are lacking. The vaccines are licensed for women between 9 and 26 years old, but may be extended to cover older women in the near future. A three-dose course costs about 3500 HKD (ca. 450 USD).

The Hong Kong department of health launched a cervical screening program since 2004 and up till December 2008; over 341000 women had registered with this program. The program mainly covers women between the ages of 25 and 64 and involves annual cervical smear for 2 years. If these are normal, smears with 3 yearly intervals would be done 
until the age of 65 years. Cervical smears can also be done for women over the age of 65 if they have never had a smear done. With this screening program in place, in 2008, about $63 \%$ of the target population had a cervical smear done within 3 years. Apart from the department of health clinics, women may also obtain smears from family planning associations, various women health clinics, as well as private family physicians and gynaecologists. The cost of a smear ranges from $100 \mathrm{HKD}$ (ca. $12.8 \mathrm{USD}$ ) to $1000 \mathrm{HKD}$. Liquidbased cytology is used in about $70 \%$ of cases.

HPV DNA testing is widely available. The Hong Kong College of O\&G guidelines recommends that it should be an adjunct to the cytology screening rather than as a primary screening test. Women who are negative by both tests can consider a longer screening interval, for example, 5-6 years.

9.3.2. Ideal Situation in Low- and High-Resource Setting. In low-resource settings, the ideal situation would be primary prevention of cervical cancer by a national vaccination program that provides a vaccine which confers the maximum protection from cervical cancer (e.g., potential multivalent vaccine). This would only be feasible if there is significant price reduction and substantial financial support from funding agencies, for example, GAVI. Once an HPV vaccine becomes affordable, the vaccines can be incorporated into school vaccination programs or into the existing Programs on Immunization.

Meanwhile, a single-visit VIA followed by cryotherapy appeared to be the most effective and feasible method. This should cover women between the ages of 25 and 49. For menopausal women, where VIA would not be suitable, there may be a role for HPV testing (e.g., single-visit HPV test + cryotherapy if HPV positive) but this would require further evidence.

Hong Kong is a region of high-resource settings, where a national HPV vaccination program should be implemented and a school vaccination program for girls aged 11-12 years appears to be an effective prevention method. Since the existing vaccine protects against $70 \%$ of all cervical cancer cases, a well-organized national screening program will still be needed. Liquid-based cytology would allow a computerized screening system, where the pathologists can concentrate on the slides most likely to contain abnormalities, as well as confirmatory (reflex) HPV testing (see Figure 4). In countries with well-established cytology screening programs, the role of HPV testing may be towards an ancillary screening test, for example, for triaging women with ASCUS. Considerations should be given to developing HPV testing as a primary screening method, especially where cytology screening is not well established.

\subsubsection{Challenges and Suggestions to Overcome the Obstacles to Cervical Cancer Prevention in Hong Kong}

(a) Economic Challenges. The lack of government funding represents the major challenge for implementation of effective vaccination against cervical cancer. However, the relative high cost of the vaccines also represents an obstacle to be overcome, even in wealthy places as Hong Kong. There is a need for multilateral negotiations involving government procurement agencies, health insurance companies, and vaccine manufacturers to optimize vaccine price and reimbursement schemes to facilitate vaccine adoption and uptake. Creating innovative partnerships among health providers to introduce school-based vaccination programs, and creating attractive self-financing schemes could contribute to lowering the costs of vaccination strategies.

(b) Education. Acceptance of HPV vaccination for adolescent girls is still low, and lack of awareness and a certain degree of anxiety among women regarding HPV and sexually transmitted infections (STI) are also obstacles to better disease prevention. Educational campaigns to inform the public about the benefits of HPV vaccination and screening are needed. In this respect, educational methods and materials adapted for different target age groups and to different socioeconomic layers of the populations should be developed, for instance, an array of educational booklets, posters, videos, or cartoons to target younger and older generations. An active effort to destigmatize the disease should be made in form of public patient testimonybased campaigns, supported by public celebrities or opinion leaders in mass media such as posters, TV, or radio spots, that may facilitate the task of making vaccination and screening popular approaches to cervical cancer prevention.

(c) Innovative Screening Strategies. Despite a 63\% coverage of the target population by the cervical screening program, a substantial subset of the population does not participate in screening and remains still at risk of inadvertently progressing to disease. In addition, for women with abnormal screening results, the waiting time for followup by colposcopy and eventually treatment are too long, reducing compliance rates to follow up and decreasing the efficiency of the program. To improve the cervical screening program, one should increase the number of clinics that can provide smear taking, especially in more "isolated areas" or outlying islands. Further, there is a need for continued training of nurses to take smears and perform colposcopy in order to have sufficient staffing and shorten the waiting time of follow-up procedures. One should also intensify "outreach" programs to inform specific populations to join current screening programs, and continue efforts to destigmatize the disease by the mass media (as stated above).

\subsection{India}

9.4.1. Current Situation in Cervical Cancer Control and Prevention in India. Cervical cancer is the leading cause of cancer-related morbidity and mortality in females. In India, there were an estimated 132,000 cases of cervix cancer in the year 2002 and 74,100 patients died of the disease [64]. The figures were generated from 18 population-based cancer registries, most of which are located in the urban areas. While there has been a gradual decline in cervical cancer incidence, 
the age-standardised rate still remains one of the highest in Asia Oceania.

Awareness of cervical cancer, as well as of HPV, is low among the population.

There is no population-based national screening program for cervical cancer [65]. Cytology-based screening continues to be opportunistic with low coverage and has not reduced the cervical cancer burden.

Recently, screening by VIA has been introduced in some low-resource settings following the demonstration of its success in research programs. The HPV DNA test is now available in the metro cities but is expensive, and awareness regarding its utility is still not universal.

Both the HPV vaccines have been licensed in India since 2008-2009, and are indicated for prevention among women between the ages of 10 to 45 years old. The vaccines are available at most private clinics and medical practitioners, and the cost of a dose was around USD 60 in the beginning but already has been reduced to USD 40 per dose.

\subsubsection{Ideal Situation in Low- and High-Resources Setting.} India is a country with varied scenarios. The majority of the population lives in rural areas in low-resource settings. This area is catered by a well laid out system of primary health centres and district hospitals, but these suffer from frequent problems of staffing, lack of resources, and so forth. About one-fourth of the population in urban areas is affluent and lives in high-resource settings. Many of these people have access to insurance and several of them have incomes that allow them to spend well on healthcare. Thus solutions need to be adapted according to the region, and within each region, to the individual setting.

Cervical cytology has been the cornerstone of cervical cancer prevention programs globally. In developed countries, widespread cytology-based screening has reduced invasive cancer cervix rates by $74 \%$ [66]. However, Pap smear has moderate sensitivity, about 50\%, although it has high specificity ( $86 \%$ to $100 \%$ ) [67]. Successful implementation requires frequent screening. Such compliance, coverage, and quality have not been feasible in India.

Overall, the recommended screening guidelines in India are as follows.

(1) Women 30-55 years old should undergo VIA screening at least once every 8-10 years as the primary screening method at rural primary health centres and subcentres and district hospitals with no Pap smear capability.

(2) VIA can be used as a screening method even in larger tertiary level hospital with limited Pap smear facilities in order to provide wider coverage to the population.

(3) VIA can be used as a triage method to guide biopsies in case of abnormal Pap smear where colposcopy facilities are not available, and Pap smear can be used to triage VIA positive cases. This will decrease the number of cases that need to be referred for further management.
(4) Positive or suspicious lesions noted upon screening should be biopsied/referred immediately.

(5) If the HPV test is available, it can be used as the primary screening method, with or without concomitant Pap smear.

(6) The availability of the rapid, affordable HPV test may open up the possibility of self-sampling for remote areas as well as for women who are reluctant to get examined.

(7) Tertiary level hospitals and cancer centres should be equipped for the management of invasive cancer. Supportive care facilities should also be available at these centres.

\subsubsection{Challenges and Suggestions to Overcome the Obstacles to Cervical Cancer Prevention in India}

\section{(a) Challenges and Suggestions in Low-Resource Settings}

(1) Political barriers

(i) There is a lack of priority for cervical cancer among public health programs, especially prevention and early detection. Health authorities and health professionals need to agree on strategies to make optimal use of limited resources.

(ii) Efforts need to be made to incorporate screening for cervical cancer into the existing primary health care facilities, in order to minimize incremental costs for the public.

(iii) Healthcare organizations representing professionals, patients, or the public in general are well positioned and empowered to call the attention of political leaders to the matter: this can be achieved by efforts to educate the population by use of mass media (television, radio, newspaper, campaigns).

(2) Community and individual barriers

(i) Among the public at large, particularly women, the lack of awareness of cervical cancer as a health problem is the major obstacle to high adherence to health programs on prevention, and compliance with screening. Some culturally specific attitudes, misconceptions and beliefs, and shyness of women inhibit discussing diseases of genital tract, and participation in screening programs. Community leaders need to be more sensitive and proactive with regard to women's health and reproductive health issues.

(ii) Awareness must be created among the healthcare professionals regarding the magnitude of the problem and the recent technological developments in the field. This may be done through educational and training programs. 
(3) Technical and organizational barriers

(i) These include poorly organized programs covering the populations at need, lack of screening registries, weak infrastructure including limited laboratory facilities, lack of trained manpower, and lack of quality control for laboratories and cytology reporting.

(ii) Protocols for screening using the methods described above should be developed and guidelines formulated for the target age women to be screened, frequency of screening, the test to be used, the reporting of the test, and the management of screen-positive women.

(iii) The number of facilities should be increased in proportion to the size of the population.

\section{(4) Cost barriers.}

VIA performed by health workers followed by cryotherapy at the same visit is associated with considerably lower costs (517 dollars/year of life saved), compared to other screening strategies [68]. There will be a cost outlay for providing some basic equipment and materials for tests, providing recurring supplies, salaries of health workers, and so forth. HPV testing, even when done only once in a lifetime, is presently costly (although cost may work out to be less than repeated screening with Pap).

Efforts need to be made to work through GAVI and other agencies to procure the HPV vaccine and to make it available through the Expanded Program on Immunization. In general, Indians accept vaccination very positively and it is expected that there will be good coverage with the HPV vaccine if it is promoted through a national program.

(b) Challenges and Suggestions in High-Resource Settings. Where resources are not a problem, there are certain other issues such as the lack of disease awareness in the community. Considerable logistic difficulties in restructuring the existing screening programs from cytology-based to HPV-based testing is a major hurdle for change.

(i) Loss to followup is also a problem even in affluent societies (although situation is better than lowresource settings). A clear policy by the government emphasizing the synergistic role of primary and secondary prevention will go a long way to improve uptake by this largely affording segment.

(ii) There is an urgent need to develop affordable HPV testing; HPV genotyping tests suitable for clinical use must be made widely available, and the healthcare providers need to be made aware of these technologies and the advantages they offer over the Pap test. HPV vaccination must be incorporated into the national routine vaccination programs.

\subsection{The Philippines}

9.5.1. Current Situation in Cervical Cancer Control and Prevention in the Philippines. The Philippines Department of Health (DOH) has advocated cervical cancer screening, but less than half (42\%) of the 389 Philippine hospitals offer screening and only $8 \%$ have dedicated screening clinics. The 2001/2002 WHO Health Survey reported a dismal 7.7\% total Pap smear coverage of Filipino women aged 18-69 years.

In February 2005, the Philippine DOH established a Cervical Cancer Screening Program to initiate an "organized" nationwide program that includes sustainable capability building, training, education, and hiring of health workers on proper VIA, Pap smear, cytology, colposcopy, and pathology. Considering low resources, VIA will be advocated as an alternative screening method for cervical cancer, especially in primary and secondary level health care facilities without Pap smear capability, by the governmental health and welfare sectors, nongovernment organizations, and professional and civil societies at the national and local levels. Pap smear with VIA triage, colposcopy, tissue biopsy, cryosurgery and surgery treatment, total abdominal hysterectomy (TAH), and total abdominal hysterectomy with bilateral salpingoophorectomy will be available at the secondary healthcare levels, plus radiotherapy and chemotherapy at the tertiary level health care facilities.

9.5.2. Ideal Situation in Low-and High-Resource Settings. The recommended screening guidelines in The Philippines are the following.

(1) Women 25-55 years old should undergo VIA (with acetic acid wash) cervical cancer screening at least once every 5 years in areas with no Pap smear capability; otherwise Pap smear will be used.

(2) Acetic acid wash (3-5\%) should be used as the primary screening method at the local health units (rural health units; health centers), district hospitals, and provincial hospitals with no Pap smear capability.

(3) VIA should be used as a triage method before Pap smear at district, provincial, and regional hospitals with Pap smear capability.

(4) Positive or suspicious lesions noted upon screening should be referred immediately.

(5) Referral centers for cervical cancer diagnostic tests and treatment should be established in tertiary facilities.

9.5.3. Challenges and Suggestions to Overcome the Obstacles to Cervical Cancer Prevention in The Philippines. The Philippine Department of Health has not formulated a policy on HPV vaccination, perhaps stemming from the most controversial concern that such formal policy could have a negative impact on the sexual behaviour of the youth. However, it may be worthwhile to consider the impressions from the Report Card-HIV Prevention for Girls and Young 
Women (The Philippines) as a framework for a prospective Philippine HPV Vaccination Program.

Financing is the major obstacle, such as a lack of political will on the part of the government to allocate the appropriate budget for the planned programs in cervical cancer screening and prevention. The lack of organized and highly detailed networking amongst the lowest levels of health care delivery may still be a major obstacle. There have been tasks initiated to begin networking through the CECAP accreditation program, but the highest level of networking with persistent and enduring coverage may yet be another task to be really accomplished.

An integrated national screening registration system that is linked to a population-based tumor registry could also be implemented to identify a cohort of vaccinated women who can be followed up and compared to unvaccinated cervical cancer cases identified from the tumor registry.

One should increase the involvement of the Philippine government officials, from the highest to the lowest rank, in the cervical cancer screening and prevention campaigns, activities, and continuing awareness. One should present the government with the challenge to increase funding and support in the activities and programs of cervical cancer screening and prevention.

\subsection{South Korea}

9.6.1. Current Situation in Cervical Cancer Control and Prevention in South Korea. In the Republic of Korea (further referred to as South Korea within this paper), urban areas are developing to high-resource settings, and on the contrary, rural areas have still insufficient, low-resource-like health care system. The incidence of cervical cancer has decreased substantially with the help of nationwide screening system. According to the Korean Central Cancer Registry, invasive cervical cancer was the fourth most common malignancy in Korean women during 1999 2001 [69], and seventh most common cancer in 2005. In consideration of age, cervical cancer is the fourth most common cancer at age of 15-34 year women and fifth most common at age of 35-64 years. The 5-year survival rate of cervical cancer between 2003 and 2007 is 80.5\%, which has raised from $77.5 \%$ in 1995 [70]. Although sexual debut in Korean women is around 21 years, later than reported in other countries [71], the prevalence of HPV in female students in urban areas is as high as 39\% [72].

For primary prevention of cervical cancer, Gardasil and Cervarix vaccine were approved for use for women in 2007 and 2008, respectively, by Korean Food and Drug Administration. A financial support system is not yet established for HPV preventive vaccination programs in Korea. An average cost for each vaccination is above 200 US\$ in Korea, so young girls are brought by parents who cover the cost financially. The vaccination guideline was announced by The Korean Society of Gynecologic Oncology and Colposcopy in 2007 [73]. Regarding screening, women 21 years and older are recommended to have a Papanicolaou smear (Pap smear) annually according to the guideline of Korean Society of Obstetrics and Gynecology. Also, women aged over 30 years can have Pap smear done for free with the support of the National Health Insurance Corporation biannually in Korea.

The Ministry of Health and Welfare reported that 55$58 \%$ of Korean women underwent Pap smear for cervical cancer program in 2005 [74]. The sensitivity of Pap smear was shown to range from $55.6 \%$ to $83.1 \%$, which was validated with the results of cervical pathology [75]. In general, it takes a few days for patients to know the result of cervical cytology taken at Korean clinics. A HPV DNA test is not involved in the primary screening system of cervical cancer and is employed as an ancillary test. In many private clinics of Korea, cervicography is popularly used as an adjunct test in the evaluation of women presenting with abnormal cytology where doctors are lacking experience with colposcopy. On the other hand, colposcopy is usually utilized at hospitals associated with medical colleges.

9.6.2. Ideal Situation in Low-Resource Settings. Unfortunately, primary prevention strategies, particularly vaccination against HPV, are not accessible to women in lowresource settings. All women 21 years and older, and until the age of 65 years, should be examined with cervical cytology by well-trained health provider every year. Cervicography can be used for women presenting with abnormal cytology results and the imaging captured by health provider is transmitted to the colposcopists through the internet or mobile instruments. Women with abnormal results of cervicography are referred to hospitals where cervical biopsy and optimal treatment can be performed.

9.6.3. Ideal Situation in High-Resource Settings. The Korean government is developing the school-based vaccination program for adolescent girls who are prior to onset of sexual debut. In general for other vaccines, the cost negotiated for vaccination is reduced and the programs are supported entirely by government funds. However, there is to date no official decision about HPV vaccination programs in Korea. In high-resource settings, Korean government has no role to play in supporting the cost of HPV vaccination for young school girls willing to get and pay for the vaccine.

9.6.4. Challenges and Suggestions to Overcome the Obstacles to Cervical Cancer Prevention in South Korea. In low-resource settings, most women are not regularly screened for cervical cancer, where health care service is not available to them, and health information is not sufficiently provided. There are few health providers able to interpret cervical cytology and provide infrastructures to store and transport the samples to laboratory safely.

(i) In low-resource settings, the most important factor to decrease the incidence of cervical cancer is likely to raise the awareness of HPV and cervical cancer, and to let women have a regular check up.

(ii) A lack of awareness of cervical cancer should be overcome by a nationwide educational program. The information about cervical cancer can be delivered through multimedia tools such as advertisement on 
television, radio, newspapers, internet, and mobile instruments. Also, mailing service from The Ministry of Health and Welfare should be started concerning the time of examination and result of cervical cytology. Regular mailing services and advertisement can compensate for loss of followup and poor compliance. Health providers are well trained and dispatched to the areas with low-resource settings.

In high-resource settings, cervical cytology should be done free for all women annually under the coordination and supervision of government. Public and private health insurance can be considered to cover the vaccination program.

\subsection{Singapore}

9.7.1. Current Situation in Cervical Cancer Control and Prevention in Singapore. Cervical cancer has been a major cancer worldwide for a long time. Countries that have successfully reduced its incidence and mortality have relied on a meticulous secondary prevention by Pap smear screening in the last 50 years. Countries which failed, or could not afford to implement a cervical screening program, continue to have a high incidence of cervical cancers.

The life expectancy of cancer patients in Singapore has increased in recent decades due to better diagnostic and screening tools as well as better treatment modalities. A steady improvement in overall long-term cancer survival was observed, and this upward trend in survival with a favourable prognosis includes cervical cancers [76].

While effective, screening and treating precancers is a very costly program to prevent cervical cancer. Unless it is maintained year after year, and indefinitely, the incidence of cervical cancer will rise again soon as the effort wanes. Hence, preventing cervical cancer will remain as a predominant health concern for as long as its preventive effort is not targeted at the principal etiologic factor and implementing primary prevention strategies.

Prophylactic HPV vaccines have been licensed in Singapore; however, routine comprehensive public vaccination program for adolescent girls has yet to be adopted by the governmental agency, despite an affirmative recommendation by medical professional bodies [77].

9.7.2. Ideal Situation in Singapore. Persistent cervical infection with high-risk oncogenic HPVs is now known to be essential etiologic factor of cervical cancers. Therefore, herein is the opportunity for the disease to be effectively controlled worldwide in a decisive manner, like how we have done so for small pox, without the need to maintain a secondary prevention system indefinitely. Effective vaccinations against most, if not all, high-risk oncogenic HPV infections must therefore be the key to potential eradication of cervical cancers.

The effectiveness of vaccination in preventing and controlling life threatening infections has been well proven in many other viral diseases like small pox, rubella, mumps, measles, poliomyelitis, Hepatitis-B, and so forth. Therefore, in a world where HPV vaccinations are given to successive cohorts of HPV-naïve women for a full generation, we will definitely see a progressive decline of cervical precancers and invasive cancers within a span of 30 to 50 years. What then is stopping the world from doing so?

\subsubsection{Challenges and Suggestions to Overcome the Obstacles} to Cervical Cancer Prevention in Singapore. Several key factors obstruct the world from adopting population HPV vaccinations to control cervical cancers effectively, namely.

(1) The expensive HPV vaccines that countries with large populations and with the higher cervical cancer rates could ill-afford.

(2) The imperfect HPV vaccines which target only 2 main oncogenic strains, albeit that they are responsible for a good majority of cervical cancers, but still not $100 \%$.

(3) The absence of governmental-sponsored cervical cancer preventive programs in many countries in the first place.

(4) The fact that cervical cancer, in a major proportion, is a disease of the poorer communities, where the monthly wage of an individual could even be less than the cost of a single dose of HPV vaccine.

Most of these obstacles are fundamentally difficult to overcome without transcending sociopolitical issues that may be highly sensitive in nature.

Firstly, there is no doubt that the solution for widespread adoption of population HPV vaccinations is that the costs of the HPV vaccines have to be very much lower than what it is today. Historically, this was the case for Hepatitis B vaccination, which was not adopted by public vaccination programs and did not become widespread until the cost of the vaccine was much reduced. Secondly, a worldwide endorsement of HPV vaccinations against cervical cancer would be most effective in reminding governmental interest, preferably by a leading health agency such as the World Health Organization. Thirdly, HPV vaccines development programs need to be expedited to produce newer generations of HPV vaccines which are more effective. Fourthly, going against the grain of current belief, it is possible that the availability of an affordable and quick cervical HPV test kit that determines a woman's cervicovaginal status of vaccinetypes HPV infection can positively impact on a woman's urge to go for HPV vaccinations. People who have been found to be at risk for hepatitis $B$, as revealed by raising suboptimal levels of Hepatitis B antibodies, would usually agree to have the vaccination, almost instantaneously. Likewise, women who are tested negative for HPV-16 and 18 may gladly see the need and the benefits of having an HPV vaccination.

The current irony of HPV vaccination is that countries which have adopted population HPV vaccination programs are countries which have an effective cervical screening program and a low incidence of cervical cancers in the first instance. These countries not only have had put cervical cancer prevention as a priority, but have now refocused their preventive effort to target the principal etiologic factor. 
This is so that one day they may be able to do away with the costly cervical screening program, when their incidences of cervical cancers become low, making population-based cervical screening programs not cost-effective to sustain. For the rest of the countries that have not even implemented an effective cervical screening program, perhaps with limited resources they may channel them to HPV vaccinations directly, hence preventing cervical cancers more effectively at the primary level.

\subsection{Taiwan}

9.8.1. Current Situation in Cervical Cancer Control and Prevention in Taiwan. The National Cervical Cancer Screening Program of Taiwan, launched in 1995, provides yearly Pap smear for women 30 years or older. The screening program includes an informative system, a quality control and monitoring system and an education system that covers public health nurses, physicians, cytotechnicians, and cytologists. Several factors support the success of this program: (1) well-established public health infrastructure, (2) highly efficient National Health Insurance Program covering 99\% of the population, and (3) highly resourced gynecological and oncology services. The registered 3-year screening rate reached 53\% coverage in 2000. From 2010, a self-sampling HPV test was provided for women who are reluctant to be screened by others. The national health statistics data before 1995 [78] and a decade after the launch of the program (2006-2007) [79], has showed a steady decline in the incidence rate of cervical cancer from 25.0 to $13.0 / 100,000$, and the mortality rate dropped from 13.8 to $5.8 / 100,000$. In fact, the mortality rate had been improving slowly and steadily before the launch of the program, mainly attributed to the continuous advancement of therapeutic care. In 2009, the overall survival rate of cervical cancer in Taiwan was $74 \%$ [80].

Several modeling studies have indicated that HPV vaccination, alone or when added to the current screening program, is cost effective in Taiwan [81, 82]. However, the consensus of mass vaccination as a major tool in cervical cancer prevention has not been completely reached. Population-based vaccination program has been running in one county and scheduled in another. A national policy of vaccinating women of low socio-economic status has been set, but not carried out yet.

9.8.2. Ideal Situation in Current Resource Setting. Although successful, the cost of cervical cancer screening is still expensive and can be much reduced by adopting new screening tools and strategies. The strategy of primary screening with HPV test with Pap smear-triage of positive cases has found sound support on both safety and effectiveness in a nationwide [83] and a large-scale regional cohort studies in Taiwan [84]. With the scenario that yearly Pap smear being applied only to women with persistent HPV infection, identified by HPV, screening every five years and a repeat test one year later for positive women, only $3 \%$ of women would require yearly Pap smear, and another 5\% may require one smear in five years. The direct and indirect costs can be cut to $35 \%$ and $23 \%$ of the current levels, respectively. Meanwhile, gene methylation test has been proved to be as sensitive as, and more specific than, HPV test in detecting CIN3 or more severe lesions in two hospital-based case-control studies in Taiwan $[85,86]$. In a nationwide multicenter cohort study, the methylation test was more effective than HPV test in triage of equivocal Pap smear results [87]. This new test holds great promise for a cytology-free, laboratory-based screening of cervical cancer and warrants further population-based studies.

9.8.3. Challenges and Suggestions to Overcome the Obstacles to Cervical Cancer Prevention in Taiwan. The procedure of cervical sampling remains to be the major psychological obstacle to women's willingness to accept cervical cancer screening in Taiwan [88]. Ideological issues such as sexuality and discrimination also exist, preventing nationwide vaccination for cervical cancer. Furthermore, the emerging success of current Pap screening program may wean the demand of the new HPV-orientated prevention tools in policy making.

With the adoption of primary HPV-oriented cytology screening the screening interval can be lengthened and at five-year interval, the cost can be largely reduced. The saved resources can be shifted to an HPV vaccination program, in a gradual and population-stratified base. The new screening strategy will require consensus and guidelines among health professionals, properly priced screening products, qualitycertified central laboratories, and most importantly, correct and convincing health messages to the public.

\subsection{Thailand}

9.9.1. Current Situation in Cervical Cancer Prevention and Control in Thailand. Thailand has long recognized the problem of cervical cancer with its highest incidence and mortality among the female cancers, with an incidence rate of 24.5 (ASR) and mortality of 12.8 per 100.000 women (cf. Globocan http://www.iarc.fr/). Many campaigns before the year 2004 for cervical cancer screening with Pap smear were not successful due to the nonsystematic approach and mostly being an opportunistic program. Screening mostly was done on the same group of women in the population and hence resulting in low coverage.

In the years 2004-2009, Thailand has established the National Screening Program using conventional Pap smear as the main method and VIA for areas of low resources. The Pap smear was widely used while the VIA was performed in only 9 out of 76 provinces. The initial goal was to have its coverage of $50 \%$ or more of the targeted population, women aged 35-60 years, at five-year interval. As a matter of fact, at the end of the first five-year period program which was in 2009, the organized program did not achieve its targeted coverage with only about $25 \%$ participation. Problem of data input may be also one of the reasons for the reported low coverage. Experience and lessons from the past five years have prompted us to reform and improve our strategy for 
the next five years period program, from 2010 to 2014, using the Pap smear all over the country, in conjunction with VIA in some areas. The program has extended the age of screening from age 30 to 60 years with a five-year interval. Funding comes from the National Health Security Office in collaboration with the management of National Cancer Institute of Thailand for data processing covering the whole country except Bangkok Metropolitan area.

9.9.2. Ideal Situation in Low- and High-Resource Settings. Primary prevention: HPV vaccines with an affordable cost for national Expanded Program on Immunization should be the ideal primary prevention strategy for young generations. A multivalent vaccine or based on the L2 capsid protein of the virus, to be administered in oral formulation (if it is possible) and temperature stable would be privileged.

Secondary prevention: Low-cost HPV test, especially with the possibility of self-collection, and equivalent sensitivity and specificity to the currently standard test, will be ideal for screening both low- and high-resource settings. Using the Pap smear as the triage and making the colposcopy with treatment of precancerous lesions available will also be an important success factor.

9.9.3. Challenges and Suggestions to Overcome the Obstacles in Cervical Cancer Prevention in Thailand. The difference of culture, educational, and socio-economical status has led some women to feel shy for pelvic examination. Vaccination would offer an alternative to overcome these cultural aspects. However, the high cost of HPV tests and vaccines has prevented these ideal methods from being widely used in Thailand. Even though some can afford for HPV testing, there is some fear of detecting the disease by a positive screening result, which is also a cause of avoiding the screening.

Misunderstanding of the principles of HPV vaccines immunization is also a cause of low vaccination adoption and coverage. Negotiations between the government and the pharmaceutical companies to provide affordable HPV tests and HPV vaccines are desirable.

The choice of using Pap smear or VIA in some areas is a dilemma. The problem of data entry from all the health service centers, with the heavy workload each center is facing, means submission of data is becoming a burden.

(i) The referral and followup system for those with abnormal result should be improved.

(ii) It is encouraged that the data on followup and cases be provided to the center for evaluation and audit.

(iii) There is a need to increase the quality of Pap smears, the number, and skills of health care personnel and cytology screeners, and for results of the test to reach the target population for followup, when applicable.

(iv) The quality of colposcopy and treatment of precancerous lesions should also improve.

(v) VIA should only be the method of choice in areas where Pap smear is not available, to avoid the confusion of the women and health care providers.
Only a comprehensive approach with coordination between the government sectors, academic and professional societies, private and charity organizations, and so forth, combined with a sustainable policy and fully support to the prevention and control of cervical cancer, will have a significant result within a short period.

\section{References}

[1] "WHO position paper on vaccines against Human Papillomavirus (HPV)," http://www.who.int/immunization/ documents/HPV_position_paper_summary.pdf.

[2] "FIGO Global Guidance for Cervical Cancer Prevention and Control,” 2009, http://www.figo.org/files/figo-corp/English _version.pdf.

[3] L. Yang, D. M. Parkin, L. Li, and Y. Chen, "Time trends in cancer mortality in China: 1987-1999," International Journal of Cancer, vol. 106, no. 5, pp. 771-783, 2003.

[4] http://data.worldbank.org/indicator/NY.GDP.MKTP.CD.

[5] A. Meheus, "Prevention of sexually transmitted infections through health education and counselling: a general framework," in New Developments in Cervical Cancer Screening and Prevention, E. L. Franco and J. Monsonego, Eds., pp. 84-90, Science, Oxford, UK, 1997.

[6] S. R. Pagliusi and M. T. Aguado, "Efficacy and other milestones for human papillomavirus vaccine introduction," Vaccine, vol. 23, no. 5, pp. 569-578, 2004.

[7] D. M. Harper, E. L. Franco, C. M. Wheeler et al., "Sustained efficacy up to 4.5 years of a bivalent L1 virus-like particle vaccine against human papillomavirus types 16 and 18 : follow-up from a randomised control trial," Lancet, vol. 367, no. 9518, pp. 1247-1255, 2006.

[8] D. R. Brown, S. K. Kjaer, K. Sigurdsson et al., "The impact of quadrivalent human papillomavirus (HPV; Types 6, 11, 16, and 18) $\mathrm{L} 1$ virus-like particle vaccine on infection and disease due to oncogenic nonvaccine HPV types in generally HPVnaive women aged 16-26 years," Journal of Infectious Diseases, vol. 199, no. 7, pp. 926-935, 2009.

[9] B. A. Slade, L. Leidel, C. Vellozzi et al., "Postlicensure safety surveillance for quadrivalent human papillomavirus recombinant vaccine," Journal of the American Medical Association, vol. 302, no. 7, pp. 750-757, 2009.

[10] A. Rowhani-Rahbar, C. Mao, J. P. Hughes et al., "Longer term efficacy of a prophylactic monovalent human papillomavirus type 16 vaccine," Vaccine, vol. 27, no. 41, pp. 5612-5619, 2009.

[11] C. K. Fairley, J. S. Hocking, L. C. Gurrin, M. Y. Chen, B. Donovan, and C. S. Bradshaw, "Rapid decline in presentations of genital warts after the implementation of a national quadrivalent human papillomavirus vaccination programme for young women," Sexually Transmitted Infections, vol. 85, no. 7, pp. 499-502, 2009.

[12] Australian Cervical Cancer Foundation, http://www.accf.org .au/.

[13] GAVI Alliance, http://www.gavialliance.org/resources/HPV_ factsheet.pdf.

[14] X. Castellsagué, A. Schneider, A. M. Kaufmann, and F. X. Bosch, "HPV vaccination against cervical cancer in women above 25 years of age: key considerations and current perspectives," Gynecologic Oncology, vol. 115, no. 3 supplement 1, pp. S15-S23, 2009. 
[15] W. Castro, J. Gage, L. Gaffikin et al., "Effectiveness, safety and acceptability of cryotherapy: a systematic literature review," January 2003, http://www.alliance-cxca.org/ english/publications.html\#cciid.

[16] R. Sankaranarayanan, B. M. Nene, S. S. Shastri et al., "HPV screening for cervical cancer in rural India," New England Journal of Medicine, vol. 360, no. 14, pp. 1385-1394, 2009.

[17] Y. L. Qiao, J. W. Sellors, P. S. Eder et al., "A new HPV-DNA test for cervical-cancer screening in developing regions: a crosssectional study of clinical accuracy in rural China," The Lancet Oncology, vol. 9, no. 10, pp. 929-936, 2008.

[18] W. Castro, J. Gage, L. Gaffikin et al., "Effectiveness, safety and acceptability of cryotherapy: a systematic literature review. Cervical Cancer Prevention: Issues in depth,” 2003, http://www.path.org/files/RH_cryo_white_paper.pdf.

[19] M. Kyrgiou, G. Koliopoulos, P. Martin-Hirsch, M. Arbyn, W. Prendiville, and E. Paraskevaidis, "Obstetric outcomes after conservative treatment for intraepithelial or early invasive cervical lesions: systematic review and meta-analysis," Lancet, vol. 367, no. 9509, pp. 489-498, 2006.

[20] R. Biellik, C. Levin, E. Mugisha et al., "Health systems and immunization financing for human papillomavirus vaccine introduction in low-resource settings," Vaccine, vol. 27, no. 44, pp. 6203-6209, 2009.

[21] L. Brabin, S. A. Roberts, R. Stretch et al., "Uptake of first two doses of human papillomavirus vaccine by adolescent schoolgirls in Manchester: prospective cohort study," BMJ, vol. 336, no. 7652, pp. 1056-1058, 2008.

[22] S. M. Garland, J. M. L. Brotherton, S. R. Skinner et al., "Human papillomavirus and cervical cancer in Australasia and Oceania: risk-factors, epidemiology and prevention," Vaccine, vol. 26, no. 12, pp. M80-M88, 2008.

[23] L. Gafirikin, M. Lauterbach, and P. D. Blumenthal, "Performance of visual inspection with acetic acid for cervical cancer screening: a qualitative summary of evidence to date," Obstetrical and Gynecological Survey, vol. 58, no. 8, pp. 543550, 2003.

[24] R. Sankaranarayanan, S. S. Shastri, P. Basu et al., "The role of low-level magnification in visual inspection with acetic acid for the early detection of cervical neoplasia," Cancer Detection and Prevention, vol. 28, no. 5, pp. 345-351, 2004.

[25] R. Sankaranarayanan, P. Basu, R. S. Wesley et al., "Accuracy of visual screening for cervical neoplasia: results from an IARC multicentre study in India and Africa," International Journal of Cancer, vol. 110, no. 6, pp. 907-913, 2004.

[26] N. Bhatla, A. Gulati, S. R. Mathur et al., "Evaluation of cervical screening in rural North India," International Journal of Gynecology and Obstetrics, vol. 105, no. 2, pp. 145-149, 2009.

[27] P. D. Blumenthal, L. Gaffikin, S. Deganus, R. Lewis, M. Emerson, and S. Adadevoh, "Cervical cancer prevention: safety, acceptability, and feasibility of a single-visit approach in Accra, Ghana," American Journal of Obstetrics and Gynecology, vol. 196, no. 4, pp. 407.e1-407.e9, 2007.

[28] S. Luciani, M. Gonzales, S. Munoz, J. Jeronimo, and S. Robles, "Effectiveness of cryotherapy treatment for cervical intraepithelial neoplasia," International Journal of Gynecology and Obstetrics, vol. 101, no. 2, pp. 172-177, 2008.

[29] R. Sankaranarayanan, P. O. Esmy, R. Rajkumar et al., "Effect of visual screening on cervical cancer incidence and mortality in Tamil Nadu, India: a cluster-randomised trial," Lancet, vol. 370, no. 9585, pp. 398-406, 2007.

[30] E. Megevand, W. Van Wyk, B. Knight, and B. Bloch, "Can cervical cancer be prevented by a see, screen, and treat program? A pilot study," American Journal of Obstetrics and Gynecology, vol. 174, no. 3, pp. 923-928, 1996.

[31] L. Denny, L. Kuhn, M. De Souza, A. E. Pollack, W. Dupree, and T. C. Wright, "Screen-and-treat approaches for cervical cancer prevention in low-resource settings: a randomized controlled trial," Journal of the American Medical Association, vol. 294, no. 17, pp. 2173-2181, 2005.

[32] E. L. Franco, "Primary screening of cervical cancer with human papillomavirus tests," Journal of the National Cancer Institute. Monographs, no. 31, pp. 89-96, 2003.

[33] Y. K. Huang, S. L. You, C. C. Yuan et al., "Long-term outcomes of high-risk human papillomavirus infection support a long interval of cervical cancer screening," British Journal of Cancer, vol. 98, no. 5, pp. 863-869, 2008.

[34] T. C. Wright, "Cervical cancer screening in the 21st century: is it time to retire the Pap smear?" Clinical Obstetrics and Gynecology, vol. 50, no. 2, pp. 313-323, 2007.

[35] T. C. Wright Jr., M. Schiffman, D. Solomon et al., "Interim guidance for the use of human papillomavirus DNA testing as an adjunct to cervical cytology for screening," Obstetrics and gynecology, vol. 103, no. 2, pp. 304-309, 2004.

[36] C. J. L. M. Meijer, T. J. M. Helmerhorst, L. Rozendaal, J. C. Van der Linden, F. J. Voorhorst, and J. M. M. Walboomers, "HPV typing and testing in gynaecological pathology: has the time come?" Histopathology, vol. 33, no. 1, pp. 83-86, 1998.

[37] M. E. Sherman, A. T. Lorincz, D. R. Scott et al., "Baseline cytology, human papillomavirus testing, and risk for cervical neoplasia: a 10-year cohort analysis," Journal of the National Cancer Institute, vol. 95, no. 1, pp. 46-52, 2003.

[38] J. T. Cox, "Human papillomavirus testing in primary cervical screening and abnormal papanicolaou management," Obstetrical and Gynecological Survey, vol. 61, no. 6, pp. S15-S25, 2006.

[39] P. E. Castle, "The potential utility of HPV genotyping in screening and clinical management," JNCCN Journal of the National Comprehensive Cancer Network, vol. 6, no. 1, pp. 8395, 2008.

[40] L. L. Villa, R. L. R. Costa, C. A. Petta et al., "High sustained efficacy of a prophylactic quadrivalent human papillomavirus types 6/11/16/18 L1 virus-like particle vaccine through 5 years of follow-up," British Journal of Cancer, vol. 95, no. 11, pp. 1459-1466, 2006.

[41] GlaxoSmithKline Vaccine HPV-007 Study Group, "Sustained efficacy and immunogenicity of the human papillomavirus (HPV)-16/18 AS04-adjuvanted vaccine: analysis of a randomised placebo-controlled trial up to 6.4 years," Lancet, vol. 374, no. 9706, pp. 1975-1985, 2009.

[42] S. K. Kjaer, K. Sigurdsson, O. E. Iversen et al., "A pooled analysis of continued prophylactic efficacy of quadrivalent human papillomavirus (types 6/11/16/18) vaccine against high-grade cervical and external genital lesions," Cancer Prevention Research, vol. 2, no. 10, pp. 868-878, 2009.

[43] S. M. Garland, M. Hernandez-Avila, C. M. Wheeler et al., "Quadrivalent vaccine against human papillomavirus to prevent anogenital diseases," New England Journal of Medicine, vol. 356, no. 19, pp. 1928-1943, 2007, (Including letter to the editor) Garland S. M., Koutsky L.A., Human Papillomavirus vaccine, New England Journal of Medicine, vol. 357, p. 11, 2007.

[44] T. Agorastos, K. Chatzigeorgiou, J. M. L. Brotherton, and S. M. Garland, "Safety of human papillomavirus (HPV) vaccines: a review of the international experience so far," Vaccine, vol. 27, no. 52, pp. 7270-7281, 2009. 
[45] A. Sutherland, H. Izurieta, R. Ball et al., "Syncope after vaccination-United States, January 2005-July 2007," Morbidity and Mortality Weekly Report, vol. 57, no. 17, pp. 457-460, 2008.

[46] http://www.fda.gov/downloads/AdvisoryCommittees/CommitteesMeetingMaterials/BloodVaccinesandOtherBiologics/ VaccinesandRelatedBiologicalProductsAdvisoryCommittee/ UCM181371.pdf.

[47] B. A. Slade, L. Leidel, C. Vellozzi et al., "Postlicensure safety surveillance for quadrivalent human papillomavirus recombinant vaccine," Journal of the American Medical Association, vol. 302, no. 7, pp. 750-757, 2009.

[48] D. FitzSimons, A. Vorsters, K. Hoppenbrouwers, and P. Van Damme, "Prevention and control of viral hepatitis through adolescent health programmes in Europe," Vaccine, vol. 25, no. 52, pp. 8651-8659, 2007.

[49] European Cervical Cancer Association, http://www.aepcc.org/ download/documentos/publico/ECCA-HPV_Vaccination_ across_Europe-April_2009.pdf.

[50] J. Bonhoeffer, A. Bentsi-Enchill, R. T. Chen et al., "Guidelines for collection, analysis and presentation of vaccine safety data in surveillance systems," Vaccine, vol. 27, no. 16, pp. 2289 2297, 2009.

[51] T. Verstraeten, D. Descamps, M. P. David et al., "Analysis of adverse events of potential autoimmune aetiology in a large integrated safety database of AS04 adjuvanted vaccines," Vaccine, vol. 26, no. 51, pp. 6630-6638, 2008.

[52] Australian Institute of Health and Welfare 2008, "Cervical Screening in Australia 2005-2006," Cancer series no. 41 cat. No. CAN36 AIHW; Canberra AIHW.

[53] J. R. Condon Jr., B. K. Armstrong, T. Barnes, and Y. Zhao, "Cancer incidence and survival for Indigenous Australians in the Northern Territory," Australian and New Zealand Journal of Public Health, vol. 29, no. 2, pp. 123-128, 2005.

[54] A. Giuliano and J. Palefsky, "Quadrivalent HPV vaccine efficacy against male genital disease and infection," in Proceedings of the 25th International Papillomavirus Conference, Malmö, Sweden, May 2009, Abstract O01-07.

[55] J. Palefsky and A. Giuliano, "Quadrivalent HPV vaccine efficacy in men having sex with men," in Proceedings of the 25th International Papillomavirus Conference, Malmö, Sweden, May 2009, Abstract P27-01.

[56] S. M. Garland, "Prevention strategies against human papillomavirus in males," Gynecologic Oncology, vol. 117, no. 2, pp. S20-S25, 2010.

[57] S. M. Garland and M. A. Quinn, "How to manage and communicate with patients about HPV?" International Journal of Gynecology and Obstetrics, vol. 94, no. 1, pp. S106-S112, 2006.

[58] M. Giles and S. Garland, "A study of women's knowledge regarding human papillomavirus infection, cervical cancer and human papillomavirus vaccines," Australian and New Zealand Journal of Obstetrics and Gynaecology, vol. 46, no. 4, pp. 311-315, 2006.

[59] M. K. Pitts, S. J. Dyson, D. A. Rosenthal, and S. M. Garland, "Knowledge and awareness of human papillomavirus (HPV): attitudes towards HPV vaccination among a representative sample of women in Victoria, Australia," Sexual Health, vol. 4, no. 3, pp. 177-180, 2007.

[60] M. Pitts, A. Smith, S. Croy et al., "Singaporean women's knowledge of human papillomavirus (HPV) and attitudes toward HPV vaccination," Women and Health, vol. 49, no. 4, pp. 334-351, 2009.
[61] M. Pitts, A. Smith, S. Croy et al., "Singaporean men's knowledge of cervical cancer and human papillomavirus (HPV) and their attitudes towards HPV vaccination," Vaccine, vol. 27, no. 22, pp. 2989-2993, 2009.

[62] C. Wen, "China's plans to curb cervical cancer," Lancet Oncology, vol. 6, no. 3, pp. 139-141, 2005.

[63] A. J. Cagle, S. Y. Hu, J. W. Sellors et al., "Use of an expanded gold standard to estimate the accuracy of colposcopy and visual inspection with acetic acid," International Journal of Cancer, vol. 126, no. 1, pp. 156-161, 2010.

[64] J. Ferlay et al., Cancer Incidence, Mortality \& Prevalence Worldwide. IARC Cancer base No.5 Version 2.0, IARC Press, Lyon, France, 2004.

[65] "Cancer Atlas India," April 2008, http://www.canceratlasindia.org/chapter6.aspx.

[66] American Cancer Society cervical cancer screening, "What are the key statistics for cervical cancer?" http://www.cancer.org.

[67] K. Nanda, D. C. McCrory, E. R. Myers et al., "Accuracy of the papanicolaou test in screening for and follow-up of cervical cytologic abnormalities: a systematic review," Annals of Internal Medicine, vol. 132, no. 10, pp. 810-819, 2000.

[68] R. Legood, A. M. Gray, C. Mahé et al., "Screening for cervical cancer in India: How much will it cost? A trial based analysis of the cost per case detected," International Journal of Cancer, vol. 117, no. 6, pp. 981-987, 2005.

[69] H. R. Shin, Y. J. Won, K. W. Jung et al., "Nationwide cancer incidence in Korea, 1999 2001; first result using the national cancer incidence database," Cancer Research and Treatment, vol. 37, no. 6, pp. 325-331, 2005.

[70] The Ministry of Health and Welfare. National Cancer Information Center.http://www.cancer.go.kr.

[71] C. J. Kim, B. G. Kim, S. C. Kim et al., "Sexual behavior of Korean young women: preliminary study for the introducing of HPV prophylactic vaccine," Korean Journal of Gynecologic Oncology, vol. 18, pp. 209-218, 2007.

[72] H. R. Shin, S. Franceschi, S. Vaccarella et al., "Prevalence and determinants of genital infection with papillomavirus, in female and male university Students in Busan, South Korea," Journal of Infectious Diseases, vol. 190, no. 3, pp. 468-476, 2004.

[73] B. G. Kim, N. K. Lee, S. C. Kim et al., "Recommendation guideline of Korean Society of Gynecologic Oncology and Colposcopy for quadrivalent human papillomavirus vaccine," Korean Journal of Gynecologic Oncology, vol. 18, pp. 259-283, 2007.

[74] Y. T. Kim, "Current status of cervical cancer and HPV infection in Korea," Journal of Gynecologic Oncology, vol. 20, no. 1, pp. 1-7, 2009.

[75] S. J. Kim, C. K. Park, H. P. Lee et al., "Multiinstitutional study for the development of cervical cancer screening model with new cervicography, Pap smear and HPV-DNA test," Korean Journal of Gynecologic Oncology, vol. 44, pp. 911-937, 2001.

[76] G. H. Lim, C. S. Wong, K. Y. Chow, V. Bhalla, and K. S. Chia, "Trends in long-term cancer survival in Singapore: 19682002," Annals of the Academy of Medicine Singapore, vol. 38, no. 2, pp. 99-105, 2009.

[77] S. K. Tay, H. Y. S. Ngan, T. Y. Chu, A. N. Y. Cheung, and E. Hseon Tay, "Epidemiology of human papillomavirus infection and cervical cancer and future perspectives in Hong Kong, Singapore and Taiwan," Vaccine, vol. 26, no. 12, pp. M60-M70, 2008.

[78] Cervical cancer screening Registration system, 13-year report, Bureau of Health Promotion, Department of the Health, Executive Yuan, R.O.C., 2009. 
[79] Y. Y. Chen, S. L. You, C. A. Chen et al., "Effectiveness of national cervical cancer screening programme in Taiwan: 12year experiences," British Journal of Cancer, vol. 101, no. 1, pp. 174-177, 2009.

[80] S. L. Koong, A. M. Yen, and T. H. Chen, "Efficacy and cost-effectiveness of nationwide cervical cancer screening in Taiwan," Journal of medical screening, vol. 13, pp. S44-S47, 2006.

[81] R. M. Rogoza, N. Ferko, J. Bentley et al., "Optimization of primary and secondary cervical cancer prevention strategies in an era of cervical cancer vaccination: a multi-regional health economic analysis," Vaccine, vol. 26, no. 5, pp. F46-F58, 2008.

[82] P. H. Liu, F. C. Hu, P. I. Lee, S. N. Chow, C. W. Huang, and J. D. Wang, "Cost-effectiveness of human papillomavirus vaccination for prevention of cervical cancer in Taiwan," BMC Health Services Research, vol. 10, article no. 11, 2010.

[83] Y. K. Huang, S. L. You, C. C. Yuan et al., "Long-term outcomes of high-risk human papillomavirus infection support a long interval of cervical cancer screening," British Journal of Cancer, vol. 98, no. 5, pp. 863-869, 2008.

[84] A. Chao, C. J. Chang, C. H. Lai et al., "Incidence and outcome of acquisition of human papillomavirus infection in women with normal cytology - a population-based cohort study from Taiwan," International Journal of Cancer, vol. 126, no. 1, pp. 191-198, 2010.

[85] H. C. Lai, Y. W. Lin, T. H. M. Huang et al., "Identification of novel DNA methylation markers in cervical cancer," International Journal of Cancer, vol. 123, no. 1, pp. 161-167, 2008.

[86] K. H. Wang, H. W. Liu, S. R. Lin, DA. C. Ding, and T. Y. Chu, "Field methylation silencing of the protocadherin 10 gene in cervical carcinogenesis as a potential specific diagnostic test from cervical scrapings," Cancer Science, vol. 100, no. 11, pp. 2175-2180, 2009.

[87] C. J. Lin, H.-C. Lai, K. H. Wang et al., “Testing for methylated PCDH10 or WT1 is superior to the HPV test in detecting severe neoplasms (CIN3 or greater) in the triage of ASC-US smear results," American Journal of Obstetrics and Gynecology, vol. 204, no. 1, pp. 21.e1-21.e7, 2011.

[88] S. I. Hou, M. E. Fernandez, E. Baumler, G. S. Parcel, and P. H. Chen, "Correlates of cervical cancer screening among women in Taiwan," Health Care for Women International, vol. 24, no. 5, pp. 384-398, 2003. 


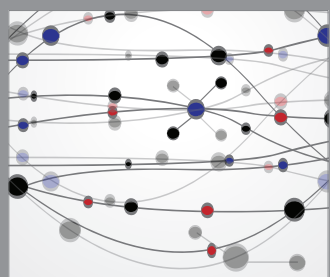

The Scientific World Journal
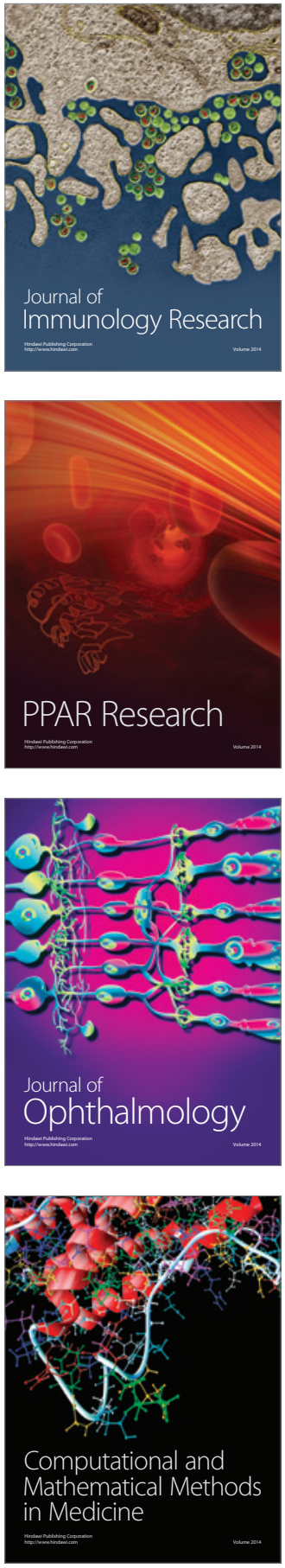

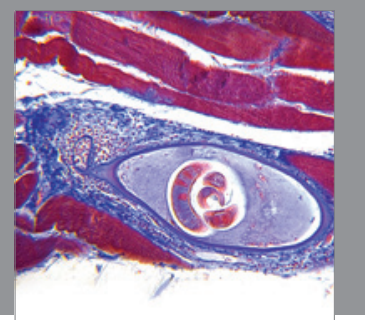

Gastroenterology

Research and Practice
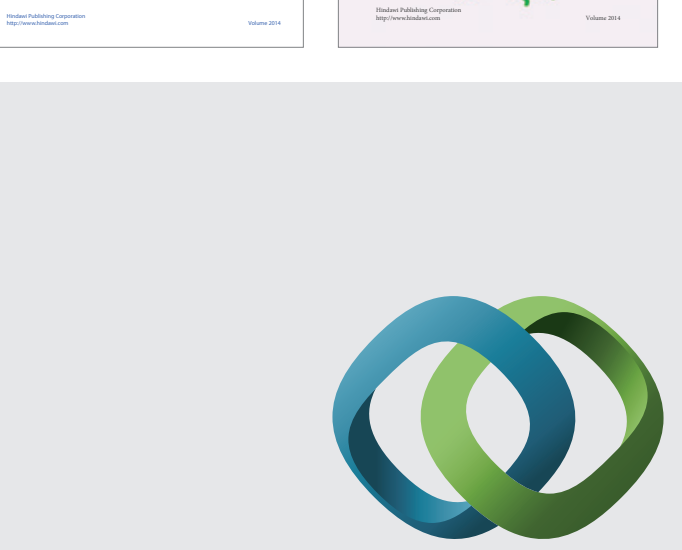

\section{Hindawi}

Submit your manuscripts at

http://www.hindawi.com
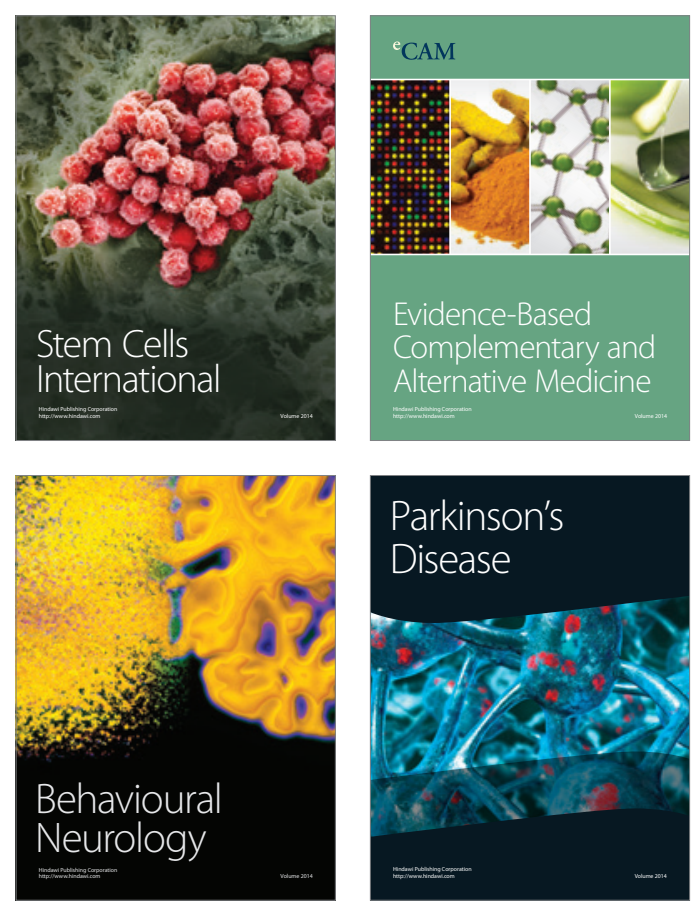

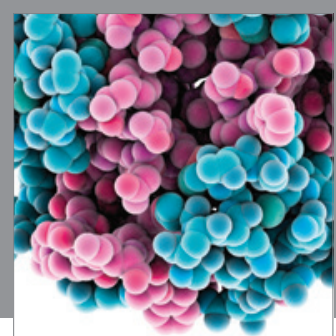

Journal of
Diabetes Research

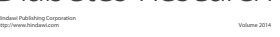

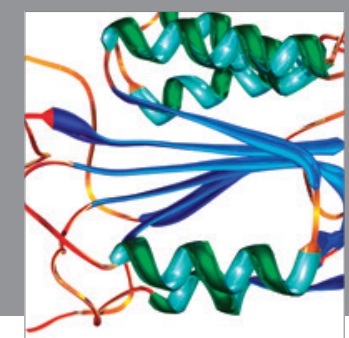

Disease Markers
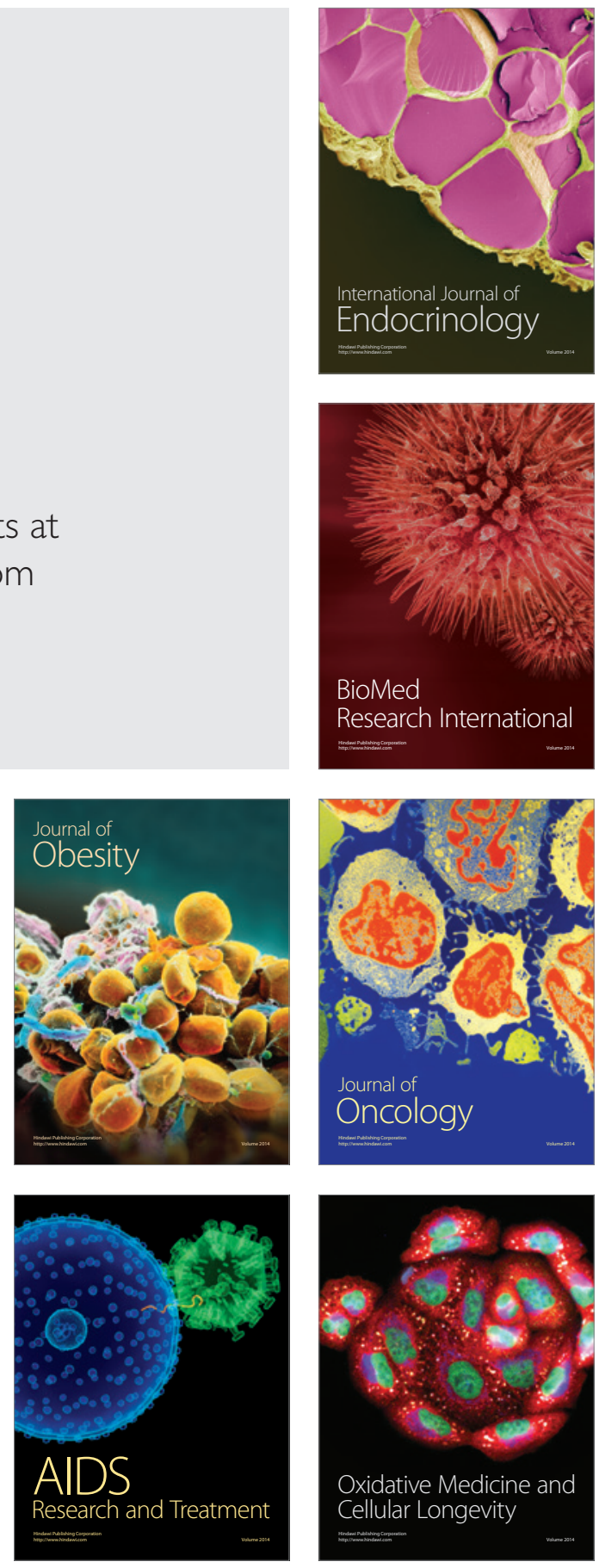